\title{
Development of a Thiol-capped Core/Shell Quantum Dot Sensor for Acetaminophen
}

\author{
Hanieh Montaseri ${ }^{\circledR}$, Oluwasesan Adegoke and Patricia B.C. Forbes* ${ }^{\star(D)}$ \\ Department of Chemistry, Faculty of Natural and Agricultural Sciences, University of Pretoria, \\ Lynnwood Road, Pretoria, 0002, South Africa
}

Received 19 November 2018, revised 24 April 2019, accepted 5 June 2019.

\begin{abstract}
Acetaminophen (AC) is a frequently used pharmaceutical which has been detected in water systems and is of concern due to its potential environmental impacts. In this study, three quantum dot (QD)-ligand systems, namely L-cysteine (L-cys)-, N-acetylL-cysteine (NAC)- and glutathione (GSH)-capped CdSe/ZnS quantum dots, were synthesized and tested for the fluorescence detection of acetaminophen. Among the synthesized aqueous core/shell quantum dots, L-cys-CdSe/ZnS QDs were found to be optimal with high sensitivity for the fluorescence detection of acetaminophen. The L-cys-CdSe/ZnS QDs were of a zinc blende crystal structure and displayed excellent fluorescence intensity and photostability and provided a photoluminescence quantum yield of $77 \%$. The fluorescence of L-cys-CdSe/ZnS QDs was enhanced by the introduction of AC enabling the development of a fast and simple method for the detection of AC. Under optimal conditions, F-F $F_{0}$ was linearly proportional to the concentration of AC from 3.0-100 nmol L ${ }^{-1}$ with limits of detection and quantification of 1.6 and $5.3 \mathrm{nmol} \mathrm{L}^{-1}$, respectively. Some related pharmaceutical compounds including epinephrine hydrochloride (EP), L-ascorbic acid (AA), uric acid (UA), dopamine hydrochloride (DA) and 4-aminophenol (4-AP) did not interfere with the sensing of AC. The probe was also successfully applied in the determination of AC in tap and river water matrices.
\end{abstract}

KEYWORDS

Acetaminophen, FRET, pharmaceuticals, quantum dots, fluorescence spectroscopy.

\section{Introduction}

Acetaminophen or paracetamol ( $\mathrm{N}$-acetyl-p-aminophenol, Fig. 1) is an acylated aromatic amide with $\log \mathrm{K}_{\mathrm{ow}} 0.46,{ }^{1}$ which was introduced in 1893 by Von Mering as antipyretic/analgesic medicine. It has a $\mathrm{pK}_{\mathrm{a}}$ of $9.46,{ }^{2}$ with a solubility of $1.4 \times 10^{4} \mathrm{mg} \mathrm{L}^{-1}$ in water at $25^{\circ} \mathrm{C} .{ }^{3}$

Acetaminophen (AC) is frequently prescribed or purchased over the counter, and falls within the pharmaceuticals and personal care products (PPCPs), a subclass of organic contaminants. Studies have shown that a large volume of pharmaceutical compounds is constantly entering the aquatic environment as well as sewers and drains from hospital waste, improper disposal of pharmaceutical waste, and from healthcare and veterinary facilities. ${ }^{4}$ In spite of the fact that the detected concentrations thereof are low, a wide variety of potential deleterious effects, including acute and chronic toxicity on the aquatic environment ${ }^{5}$ and accumulation in tissues ${ }^{6}$ have been reported even at these low levels.

In order to avoid adverse effects of PPCPs to humans, fast and sensitive analytical methods are needed for detecting low concentrations of PPCPs in water systems. AC has been reported to occur within the $6.5-11.8 \mathrm{nmol} \mathrm{L}^{-1}$ range in river waters in South Africa, ${ }^{7,8}$ while it was detected in Kenyan river waters up to $708.0 \mathrm{nmol} \mathrm{L}^{-1}$. . The concentration range of $\mathrm{AC}$ in water has been found to range from $0.86-1627 \mathrm{nmol} \mathrm{L}^{-1} 10,11$ in wastewater influent and 0.07-460 nmol L ${ }^{-11}$ in wastewater effluent.

At present, detection of PPCPs is carried out under laboratory conditions utilizing highly expensive instruments, trained personnel, environmentally unfriendly solvent use and long analysis times. Techniques include high performance liquid

* To whom correspondence should be addressed. E-mail: patricia.forbes@up.ac.za

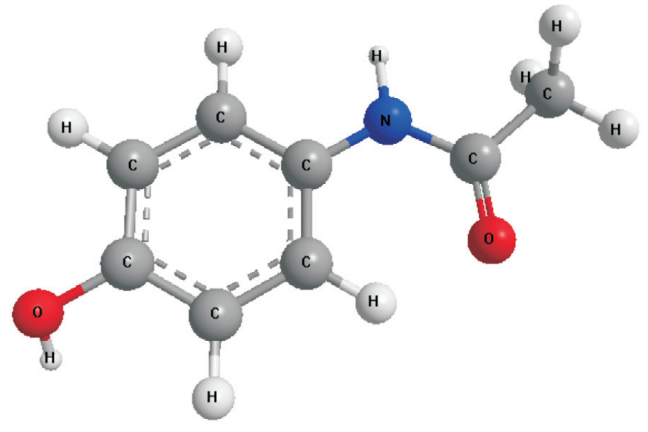

Figure 1 Structure of acetaminophen.

chromatography, ${ }_{13}^{13}$ electrochemical analysis ${ }^{14}$ spectrophotometry ${ }^{15}$ and chemiluminescence. ${ }^{16}$ To circumvent these issues, simple analytical methods are being developed to detect low concentrations of AC. Among them, optical measurement methods have gained attention due to advantages including high sensitivity, ease of implementation, and generally fast response times. ${ }^{17}$ Fluorescence techniques are a popular subset of optical methods, which use a fluorophore material as a sensing receptor to detect the analyte(s). Examples of fluorophore nanomaterials are carbon nanoparticles (CNPs), graphene quantum dots (QDs) and semiconductor QDs of the class of group II-VI and III-V. ${ }^{18}$

QDs comprise a semiconductor core and a shell which protects them against oxidation and photodegradation. In addition, QDs are photo electrochemically active which make them reliable optical labels in environmental analysis. ${ }^{19}$

Although the core QDs are toxic due to the use of Cd, stabilization thereof with an inorganic shell such as $\mathrm{ZnS}$ and also surface 
coating with a capping agent (e.g. L-cysteine or glutathione) can reduce ion leakage, thereby reducing toxicity in use. ${ }^{20}$

QD-based sensing applications are due to Förster resonance energy transfer (FRET) where QDs serve as donor, and reports of their application as acceptor are less common. The photoluminescence (PL) 'turn-on' mode is more desirable compared to the 'turn-off' mode, as many other factors can also induce the PL 'off' state resulting in false positives. ${ }^{21}$

It has been previously shown that high lattice mismatch $(\sim 16 \%)$ between $\mathrm{ZnS}$ and CdTe may cause incomplete coating of the core QDs with ZnS which makes the QDs susceptible to degradation..$^{22}$ Lower lattice mismatch $(\sim 12 \%)$ for CdSe and $\mathrm{ZnS}$ however, results in less degradation. ${ }^{23}$ Therefore, CdSe/ZnS QDs are more stable and less toxic than CdTe/ZnS QDs. Moreover, CdSe/ZnS QDs have exhibited the least cytotoxicity and the most biocompatibility with high sensing ability among CdSe, $\mathrm{CdSe} / \mathrm{ZnS}$ and CdSe/CdS QDs. ${ }^{24}$

Core/shell QDs have a number of advantageous properties regarding their use as analytical sensors and they have found many applications across disciplines. ${ }^{25,26}$

Even though AC has been detected by several analytical techniques, there is still a lack of a reliable, fast and cost-effective screening method for this analyte. Herein, three QD-thiol ligand combinations were synthesized and their sensitivity towards AC detection was examined in order to find the optimum QDligand system. We then describe a simple turn-on fluorescence detection approach for AC sensing based on L-cys-CdSe/ZnS QDs as these provided the highest sensitivity. Although many studies have been published regarding the application of $\mathrm{CdSe} / \mathrm{ZnS}$ QDs, there is no study to date regarding their use in the determination of $\mathrm{AC}$ in environmental water samples.

\section{Experimental Procedures}

\subsection{Materials}

Cadmium oxide (99.5\%), octadec-1-ene (ODE, $90 \%)$, L-cysteine (L-cys, $96 \%)$, sulfur powder $(\geq 99.5 \%)$, trioctylphosphine oxide (TOPO, $90 \%)$, zinc oxide $(99 \%)$, oleic acid (OA, $90 \%$ ), N-acetyl-L-cysteine (NAC, $\geq 99 \%$ ) and acetaminophen (AC, analytical standard) were purchased from Sigma Aldrich (St. Louis, MO, USA). Epinephrine hydrochloride (EP, $95 \%$ ), L-ascorbic acid (AA, reagent grade), dopamine hydrochloride (DA, $\geq 98 \%$ ), and uric acid (UA, $\geq 99 \%$ ) and 4-aminophenol (4-AP, $99.9 \%)$ as potential interfering analytes were also purchased from Sigma Aldrich. Methanol (99.5\%), acetone $(99.5 \%)$, sulfuric acid $(98 \%)$, potassium hydroxide $(85 \%)$, chloroform $(99 \%)$, glutathione $(\mathrm{GSH}, \geq 98 \%)$ and selenium powder $(99 \%)$ were purchased from Merck (Darmstadt, Germany). Water was provided by an ultrapure Milli-Q Water System $\left(18.0 \Omega . \mathrm{cm}\right.$ at $\left.25^{\circ} \mathrm{C}\right)$.

\subsection{Instrumentation}

A Horiba Jobin Yvon Fluoromax-4 spectrofluorometer was employed to record fluorescence emission spectra and UV-vis absorption spectra were taken using a Cary Eclipse (Varian) spectrophotometer.

The PL quantum yields of the QDs in Millipore water were determined as previously reported. ${ }^{27}$ A PANalytical $X^{\prime}$ Pert Pro powder diffractometer in $\theta-\theta$ configuration with a $X^{\prime}$ Celerator detector, variable divergence and receiving slits with Fe filtered Co-K $\alpha$ radiation $(\lambda=1.789 \AA)$ was used to analyze powder X-ray diffraction (XRD) patterns. IR measurements were obtained from a Spectrum RXI FT-IR System from Perkin Elmer. Transmission electron microscopy (TEM) images were recorded using a JEOL JEM 2100F operated at $200 \mathrm{kV}$. The particle size distributions of QDs were estimated using ImageJ software (http:// imagej.nih.gov/ij/, U.S. National Institutes of Health [NIH], Bethesda, Maryland, USA). Raman spectra were recorded using a WITec Alpha 300 micro-Raman imaging system with $488 \mathrm{~nm}$ excitation laser and CCD detector at room temperature with laser power below $2 \mathrm{~mW}$ in order to minimize heating effects. All $\mathrm{pH}$ measurements were made with a Metrohm $780 \mathrm{pH}$-meter that was calibrated with Accsen standards of $\mathrm{pH} 4.0$ and 7.0. Energy dispersive X-ray spectroscopy (EDS) analysis was carried out using a HRSEM integrated EDS Zeiss Crossbeam 540 with software AZtech version 3 by Oxford Instruments. PhotochemCAD was used to compute the Förster distance. ${ }^{28}$

\subsection{Fabrication of Water-soluble CdSe/ZnS Core/Shell QDs}

A one pot organometallic approach was utilized for the preparation of the CdSe/ZnS core/shell QDs which has previously been reported by our group. ${ }^{27}$ Conversion of hydrophobic QDs to water-soluble QDs was then carried out by a ligand exchange reaction. A stock solution of thiol ligand- $\mathrm{KOH}$ in $40 \mathrm{~mL}$ methanol was prepared using $2 \mathrm{~g}$ of thiol ligand (L-cys, GSH or NAC) and $3.0 \mathrm{~g}$ of $\mathrm{KOH}$ with ultrasonication. After purification, the hydrophobic capped CdSe and CdSe/ZnS QDs were re-dispersed separately in chloroform and the ligand exchange solution was added followed by Millipore water. In order to separate the organic phase from the aqueous phase, the solutions were stirred for $1 \mathrm{~h}$. The water-soluble CdSe and CdSe/ZnS QDs were then purified using ethanol and acetone to efficiently remove all hydrophobic capping substituents. The purified water-soluble QDs were finally dried in a fume hood.

\subsection{Fluorescence Experiments}

$1.0 \mathrm{mg}$ of water-soluble CdSe/ZnS QDs was dissolved in $3 \mathrm{~mL}$ Millipore water and a standard solution containing a specific amount of AC was added and after 5 min the resulting PL spectrum was determined. The same conditions were applied for all PL measurements: $5 \mathrm{~nm}$ slit width was chosen for the excitation and emission of the spectrofluorometer with a $300 \mathrm{~nm}$ excitation wavelength. An AC stock solution $\left(1.4 \times 10^{-3} \mathrm{~mol} \mathrm{~L}^{-1}\right)$ was prepared by dissolution of the solid in Millipore water and was diluted to prepare standard solutions. Sensing was carried out as follows: $2 \mathrm{~mL}$ of QDs solution was placed in a quartz cuvette followed by addition of $500 \mu \mathrm{L}$ of AC standard solution. The PL intensity was taken after 5 min as $F$ and $F_{0}$ was PL intensity of QDs in $500 \mu \mathrm{L}$ of water. A series of $\mathrm{F}-\mathrm{F}_{0}$ values were obtained by varying the AC concentration.

Real water samples for fluorescence measurements were collected in pre-cleaned glass bottles from a river and municipal tap in Pretoria, South Africa, and were transported to the laboratory in a cooler box filled with icepacks. The water samples were centrifuged at $4500 \mathrm{rpm}$ for $5 \mathrm{~min}$ and filtered using $110 \mathrm{~mm}$ pore size filter paper prior to analysis.

\section{Result and Discussion}

\subsection{Comparison of PL Intensity of L-cys-CdSe/ZnS,} NAC-CdSe/ZnS and GSH-CdSe/ZnS QDs

The synthesized CdSe/ZnS QDs capped with three different capping agents, L-cys, NAC and GSH, respectively, were utilized to investigate the optimum QD-ligand system for the determination of AC. The relative fluorescence intensity of the QDligand combinations was measured by the amount of enhancement observed for $5.0 \mathrm{nmol} \mathrm{L}^{-1}$ of AC relative to the fluorescence signal of the QDs in the presence of Millipore water. It was observed that the NAC-capped CdSe/ZnS QDs provided the 
lowest sensitivity while the fluorescence intensities of the L-cys and GSH-CdSe/ZnS QDs were easily influenced by the addition of $A C$ and showed greater sensitivity (Fig. 2). There was a $23 \%$ increase in the relative PL intensity of the L-cys-capped core/shell QDs while only an increase of $2 \%$ was found for the NAC-capped QDs and $21 \%$ for the GSH-capped QDs. In contrast, GSH-CdSe/ZnS QDs were previously found to provide sensitive detection of triclosan by our group. ${ }^{27}$ In this case, L-cys-CdSe/ZnS QDs provided the most sensitivity and were thus chosen as a fluorescent sensor for the determination of AC and all subsequent characterizations and experiments were performed on L-cys-CdSe/ZnS core/shell QDs.

In a previous study, ${ }^{27}$ the Förster distance was found to be $4.0 \mathrm{~nm}$ for the system of GSH-CdSe/ZnS QDs-TCS while this distance in the case of L-Cys- and NAC-CdSe/ZnS QDs for TCS was $7.9 \mathrm{~nm}$ and $7.8 \mathrm{~nm}$, respectively. Therefore, GSH-CdSe/ZnS QDs provided higher sensitivity with better energy transfer. In this study, the calculated Förster distance for both L-cys- and NAC-CdSe/ZnS QDs for AC target molecules were $6.1 \mathrm{~nm}$ (the Förster distance of L-cys-CdSe/ZnS QDs-AC system is discussed in Section 3.11). It was therefore concluded that the lower sensitivity of NAC-CdSe/ZnS QDs arose most likely from the structure of NAC which could not efficiently H-bond to AC molecules relative to L-cys for which three $\mathrm{H}$-bonds are possible.

\subsection{Optical Characterization of Aqueous L-cys-CdSe and L-cys-CdSe/ZnS QDs}

An overlay of the emission and absorption spectra of core and core/shell QDs is shown in Fig. S1. where a characteristic broad absorption and narrow emission peak are evident. Table 1 compares the optical parameters of the water-soluble core and core-shell QDs.
Surface passivation with the $\mathrm{ZnS}$ shell increased the size of the core-shell QDs, as evident from the red shift. Stokes shift is one of the most important parameters of semiconductor QDs for the elucidation of their optical properties which is known as the red shift of the emission spectra in respect of the absorption spectra. ${ }^{29}$ Accordingly, the Stokes shift of L-cys-CdSe and L-cys-CdSe/ZnS QDs were determined to be $30 \mathrm{~nm}$ and $15 \mathrm{~nm}$, respectively. The significant fluorescence enhancement of $\mathrm{CdSe} / \mathrm{ZnS}$ QDs is a result of the influence of the larger band gap induced by the $\mathrm{ZnS}$ shell which efficiently traps the electrons in the core, and also due to the removal of dangling surface bonds in the QD core upon addition of the shell (Scheme S1). ${ }^{30}$

The extinction coefficient of L-cys-CdSe and L-cys-CdSe/ZnS QDs were calculated according to a published method. ${ }^{31}$ The concentrations of L-cys-CdSe and L-cys-CdSe/ZnS QDs in Millipore water can be therefore calculated from the Beer-Lambert law.

The calculated emission band gap values for the synthesized QDs were calculated to be 2.18 and $2.09 \mathrm{~nm}$ for L-cys-CdSe and L-cys-CdSe/ZnS QDs, respectively. The PLQY of core/shell QDs increased dramatically due to the passivation of surface vacancies and non-radiative recombination sites after coating with the $\mathrm{ZnS}$ shell..$^{32} \mathrm{~A}$ red shift of $26 \mathrm{~nm}$ was observed in the PL emission of L-cys-CdSe/ZnS QDs in comparison to the L-cys-CdSe core at the optimum excitation wavelengths, which is due to the formation of the shell layer. The strong PL band with narrow FWHM of L-cys-CdSe/ZnS QDs provides evidence for the formation of a uniform particle distribution. ${ }^{24}$

\subsection{Structural and Composition Characterization of L-cys-capped Core/Shell QDs}

EDS analysis was performed to prove the presence of the metal

Table 1 Optical characterization values of the water-soluble L-cys-CdSe and L-cys-CdSe/ZnS QDs.

\begin{tabular}{|c|c|c|c|c|c|c|c|}
\hline QDs & $\begin{array}{l}\text { Excitonic peak } \\
\qquad / \mathrm{nm}\end{array}$ & $\begin{array}{l}\text { PL peak } \\
/ \mathrm{nm}\end{array}$ & $\begin{array}{l}\text { Extinction coefficient } \\
\qquad / \mathrm{L} \mathrm{mol}^{-1} \mathrm{~cm}^{-1}\end{array}$ & $\begin{array}{l}\text { PLQY } \\
1 \%\end{array}$ & $\begin{array}{l}\text { Stokes shift } \\
\quad / \mathrm{nm}\end{array}$ & $\begin{array}{l}\mathrm{FWHM} \\
/ \mathrm{nm}\end{array}$ & $\begin{array}{l}\text { Calculated band gap } \\
\text { energy value/eV }\end{array}$ \\
\hline L-cys-CdSe QDs & $\begin{array}{c}460 \\
\text { (shoulder) } \\
539\end{array}$ & 569 & $9.3 \times 10^{4}$ & 5.4 & 30 & 40.0 & 2.18 \\
\hline L-cys-CdSe/ZnS QDs & 580 & 595 & $2.04 \times 10^{5}$ & 77 & 15 & 38.5 & 2.09 \\
\hline
\end{tabular}

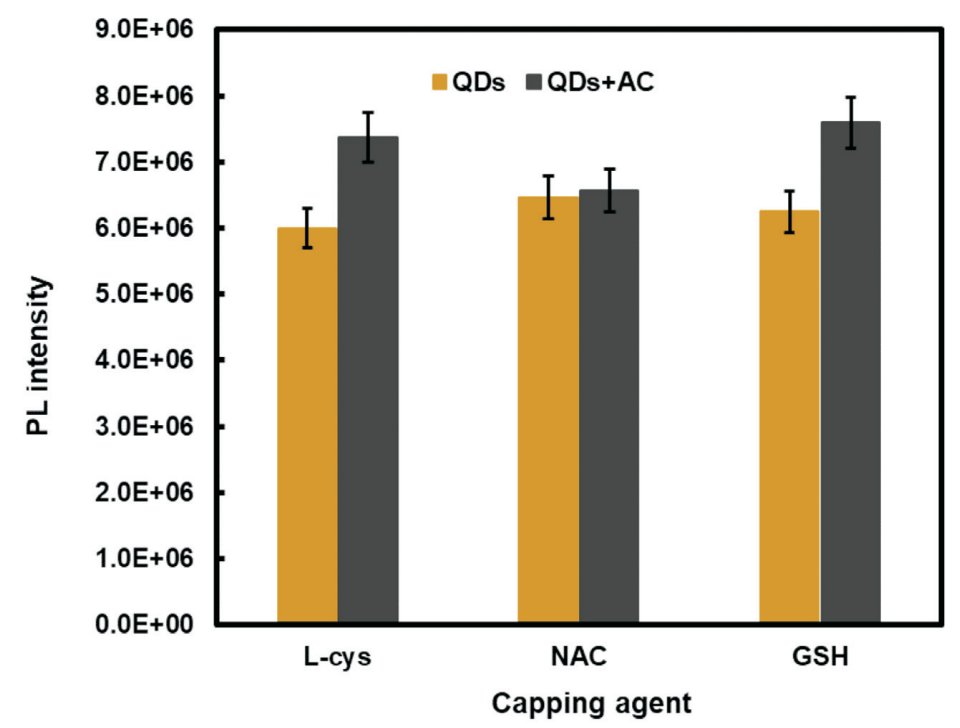

Figure 2 Comparison of the sensitivity of three QD-ligand systems (L-cys-CdSe/ZnS, NAC-CdSe/ZnS and GSH-CdSe/ZnS QDs) towards 5.0 nmol L-1 of AC. 
components in the structure of the L-cys-CdSe and L-cys-CdSe/ $\mathrm{ZnS}$ QDs (Fig. S2). The signals of $\mathrm{Cd}$ and Se were found in the core while the presence of $\mathrm{Zn}$ and $\mathrm{S}$ in the core/shell QDs confirmed the addition of the $\mathrm{ZnS}$ shell on the core. In the EDS spectra additional $\mathrm{C}, \mathrm{N}$ and $\mathrm{O}$ signals arose from the capping ligand L-cys. For these core QDs, the capping agent also resulted in a $S$ signal.

The X-ray diffraction patterns of L-cys-CdSe and L-cys-CdSe/ ZnS QDs were compared. Fig. S3 indicates a typical zinc blende crystal structure with planes at $\{111\} ; 2 \theta=31.56,\{220\} ; 2 \theta=$ 52.71 and $\{311\} ; 2 \theta=62.74$ for $\mathrm{CdSe} / \mathrm{ZnS}$ QDs. The peaks for CdSe QDs were at $29.57^{\circ}, 49.18^{\circ}$ and $58.44^{\circ}$ corresponding to $\{111\},\{220\}$ and $\{311\}$ according to the standard JCPDS (Card No.19-0191) of bulk cubic CdSe. The diffraction peaks for the CdSe/ZnS QDs were shifted slightly to higher Bragg angles compared to the CdSe core. The broadening of the diffraction peak can also be attributed to the nano-sized dimensions of the nanocrystals. ${ }^{33}$ The zinc blende crystal structure was still predominant for CdSe QDs but with weaker diffraction peaks compared to CdSe/ZnS QDs.

FT-IR analysis allowed for the characterization of the functional groups on the surface of the QDs (Fig. 3). Functional groups and IR absorption bands of L-cys-CdSe and L-cysCdSe/ZnS QDs are summarized in Table S1. Carboxylic acid and amino groups were found on the surface of the L-cys capped CdSe/ZnS and CdSe QDs while the S-H group vibration was absent on the surface of the QDs, which is attributed to the formation of $\mathrm{ZnS}$ onto the surface of the core, and core/shell QDs.

\subsection{Raman Analysis}

The Raman spectra of L-cys CdSe and L-cys-CdSe/ZnS QDs are shown in Fig. S4. Generally, nanomaterials with small dimensions from group II-VI show broad spectra. The Raman lines of the CdSe core illustrate longitudinal optical LO and 2LO phonons in the region of $200 \mathrm{~cm}^{-1}$ and $400 \mathrm{~cm}^{-1}$, respectively while a weaker mode of the second-order transverse acoustic (TA) appeared at around $103 \mathrm{~cm}^{-1} \cdot{ }^{34}$ This multi phonon scattering in semiconductor QDs is characteristic of the resonantly excited Raman processes. ${ }^{35}$ The Raman lines of $\mathrm{LO}$ and $2 \mathrm{LO}$ phonons in CdSe/ZnS QDs are located at around $190.5 \mathrm{~cm}^{-1}$ and $577 \mathrm{~cm}^{-1}$, respectively. Moreover, the LO line of the $\mathrm{ZnS}$ shell was observed at about $300 \mathrm{~cm}^{-1}$. The D-band at $1350 \mathrm{~cm}^{-1}$ confirms the presence of disorder in $\mathrm{sp}^{2}$-hybridized carbon systems and the G-band $\left(1580 \mathrm{~cm}^{-1}\right)$ arises from the stretching of the C-C bond of all pairs of $\mathrm{sp}^{2}$-hybridized carbon atoms in both the rings and chains, ${ }^{36}$ which are evidence of the presence of graphitic carbon from L-cys.

\subsection{Comparison of Particle Size of the Water-soluble \\ L-cys-CdSe/ZnS QDs}

The particle size of L-cys-capped core and core/shell QDs were estimated by using three approaches including first excitonic absorption peak, XRD and TEM (Table 2).

The particle size of the L-cys-CdSe and L-cys-CdSe/ZnS QDs determined from the first excitonic absorption peak using a published method ${ }^{31}$ and the average crystallite size of the L-cys-CdSe and L-cys-CdSe/ZnS QDs were estimated from the Debye Scherrer's equation. ${ }^{37}$ The average particle size of the core/shell QDs shows that each particle of the synthesized L-cys-CdSe/ZnS QDs consists of two or more individual crystallites.

In addition, the average particle size distributions \pm standard deviation of the QDs were estimated to be $2.1 \pm 0.5$ and $5.1 \pm$

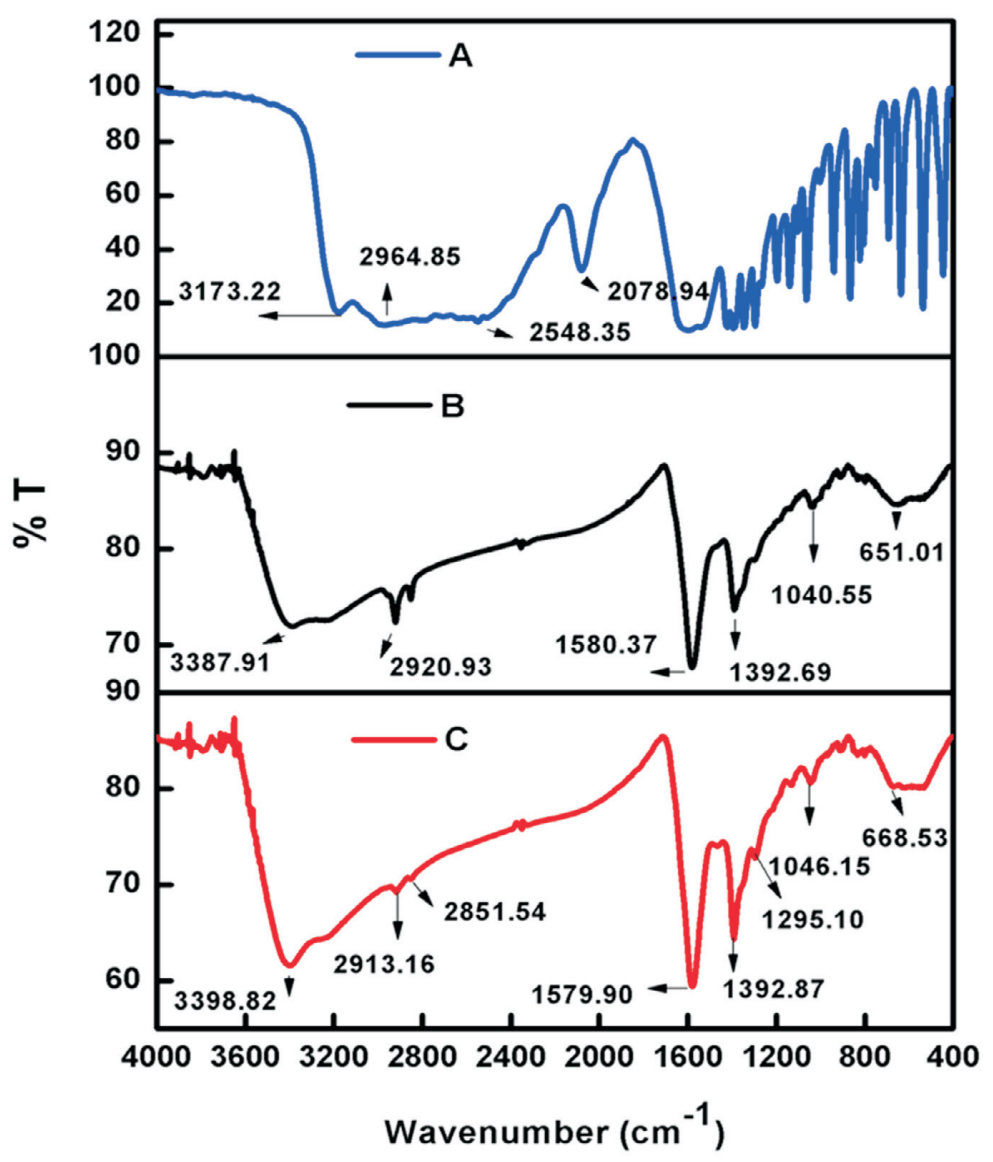

Figure 3 The FT-IR spectra of (A) L-cys, (B) L-cys-CdSe QDs and (C) L-cys-CdSe/ZnS QDs. 
Table 2 Comparison of the particle size of L-cys-CdSe and L-cys-CdSe/ ZnS QDs.

\begin{tabular}{lcc}
\hline Method & $\begin{array}{c}\text { L-cys-CdSe QDs } \\
/ \mathrm{nm}\end{array}$ & $\begin{array}{c}\text { L-cys-CdSe/ZnS QDs } \\
/ \mathrm{nm}\end{array}$ \\
\hline First excitonic peak & 2.8 & 4.0 \\
XRD & 3.00 & 3.03 \\
TEM & $2.1 \pm 0.5$ & $5.1 \pm 0.8$ \\
\hline
\end{tabular}

$0.8 \mathrm{~nm}$ for L-cys-CdSe and L-cys-CdSe/ZnS QDs, respectively from the TEM micrographs, which were in agreement with the sizes estimated from the first excitonic absorption peak. As an indirect approach ${ }^{38}$ the shell thickness of $\mathrm{ZnS}$ was calculated to be $1.5 \mathrm{~nm}$. The morphological display of the QDs showed that they were nearly homogenous in nature, monodispersed and also spherical in shape (Fig. 4A,B). TEM also showed narrow particle size distributions for L-cys-CdSe and L-cys-CdSe/ZnS QDs with a standard deviations below $1 \%$ (Fig. 4A1-B1).

\subsection{Fluorescence Properties of AC}

The possible influence of AC on the PL spectrum of L-cys$\mathrm{CdSe} / \mathrm{ZnS}$ QDs was evaluated by recording its PL using different excitation wavelengths as AC itself fluoresces. The highest emission intensity was around $400 \mathrm{~nm}$ at the excitation wavelength of $300 \mathrm{~nm}$ (Fig. S5). FRET is therefore a possible mechanism for the fluorescence enhancement noted upon interaction of AC with the QDs, because there are overlapping bands between the absorption spectrum of the QDs and the emission spectrum of $A C$ in the range $350-650 \mathrm{~nm}$.

\subsection{Investigation of Photostability}

The fluorescence intensity of L-cys-CdSe/ZnS QDs in
Millipore water increased over time and became stable after $24 \mathrm{~h}$.

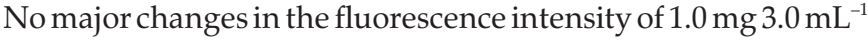
QD solutions were noted even after storage for 5 days in solution under ambient light conditions (Fig. S6) although the band gap of the L-cys-CdSe/ZnS QDs decreased which can be seen from the red shift in the PL emission of the QDs. Aggregation can induce such a red shift in the QD fluorescence which would also be accompanied by PL quenching. The slight decrease in fluorescence intensity between day 1 to day 5 may also be due to desorption of L-cys from the surface of CdSe/ZnS QDs, as L-cys acts as a surface passivating agent, which eliminates surface defects. ${ }^{39}$ Subsequent analytical measurements were thus performed after the fluorescence intensity of QDs had become stable after one day.

\subsection{Optimization of Aqueous L-cys-CdSe/ZnS QD \\ Concentration and Incubation Time}

Taking into consideration that the concentration of QDs can influence the fluorescence intensity, different concentrations of L-cys-CdSe/ZnS QDs (1.0, 1.5, 2.0, 2.5, and $3.0 \mathrm{mg}$ in $3.0 \mathrm{~mL}$ water) were prepared and $5.0 \mathrm{nmol} \mathrm{L}^{-1}$ of $\mathrm{AC}$ was added to each. Briefly, $500 \mu \mathrm{L}$ of a fixed concentration of AC was added to $2 \mathrm{~mL}$ of each concentration of QDs and the fluorescence intensity was recorded after $5 \mathrm{~min}$. It was observed that the sensitivity reduced at high concentrations of water-soluble L-cys-CdSe/ZnS QDs likely due to self-quenching or agglomeration effects, whereas very low concentrations of QDs led to a narrowing of the linear range. The optimum concentration of $1.0 \mathrm{mg}$ of L-cys-CdSe/ZnS QDs in $3.0 \mathrm{~mL}$ water was found, indicating an increase in the interaction of AC with QDs in solution (Fig. 5).

In order to determine the optimum interaction time of L-cys-CdSe/ZnS QDs with AC, the fluorescence intensity of the system was examined at different time intervals in the presence
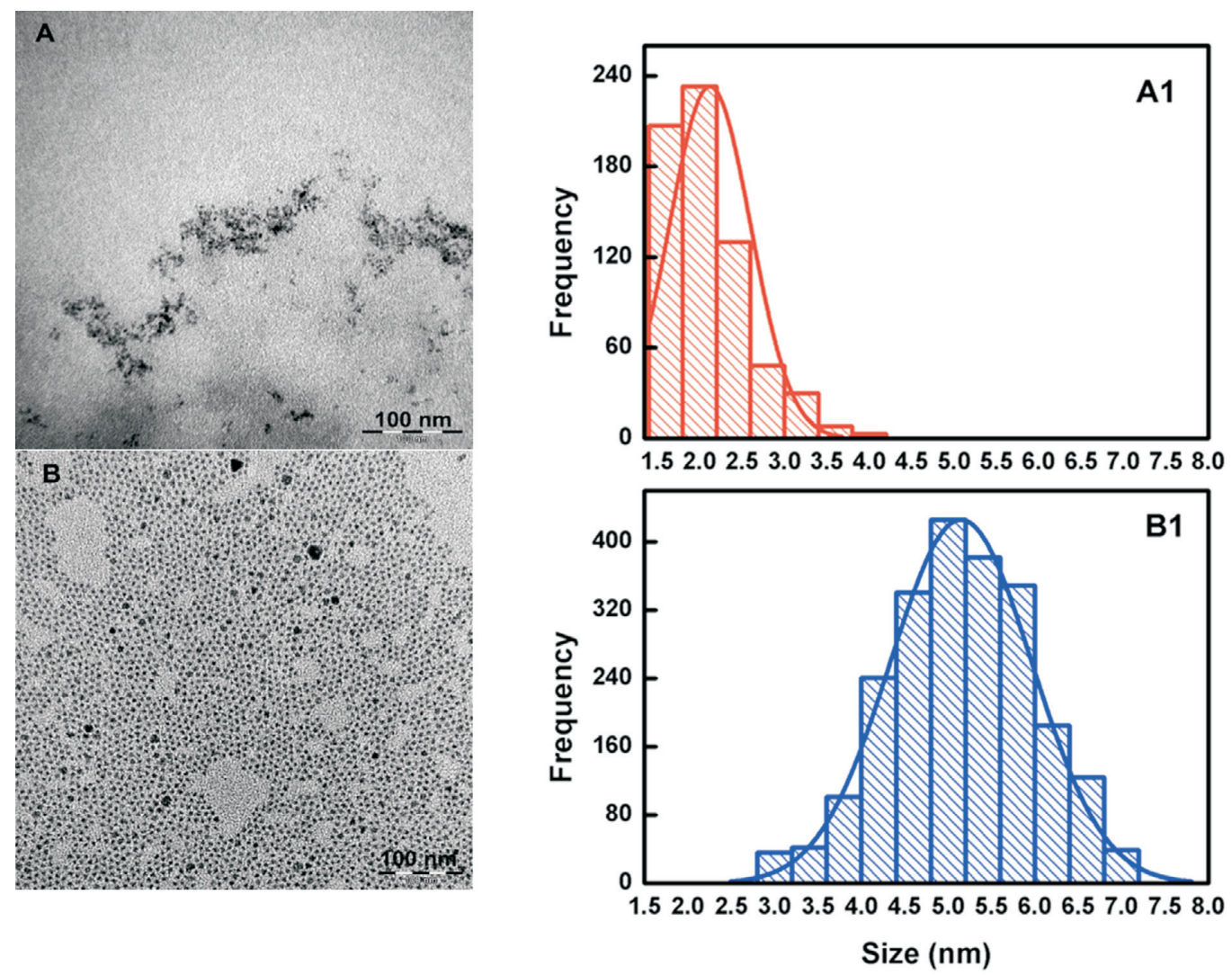

Figure 4 ( A) TEM images of L-cys-CdSe QDs and (B) L-cys-CdSe/ZnS QDs, scale bar $100 \mathrm{~nm}$; and (A1) particle size distribution of L-cys-CdSe QDs and (B1) L-cys-CdSe/ZnS QDs. 


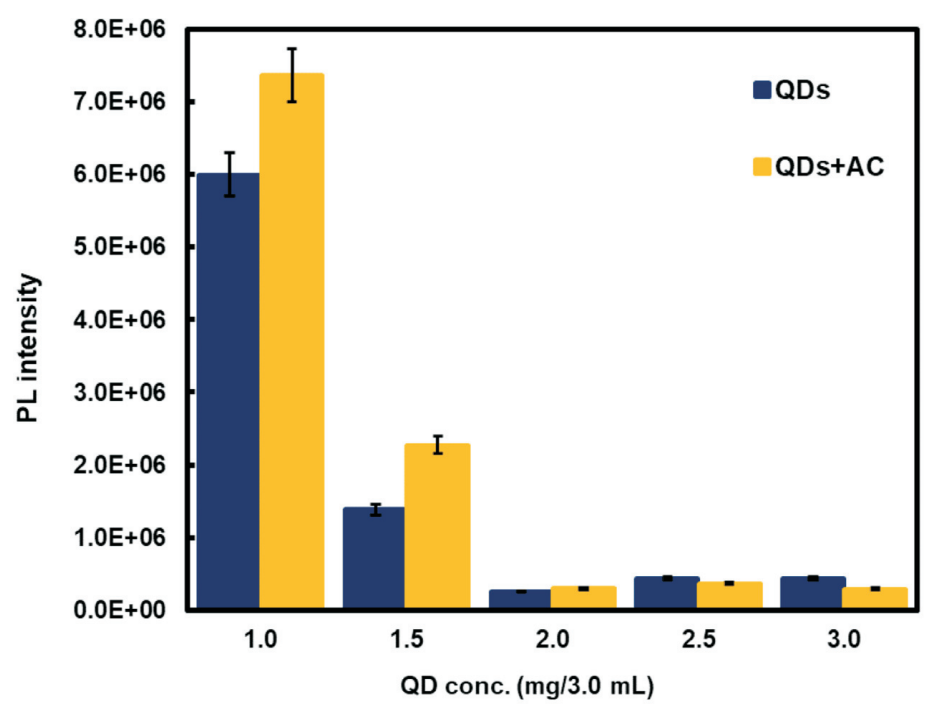

Figure 5 Effect of the concentration of aqueous L-cys-CdSe/ZnS QDs on fluorescence in the absence and presence of $5.0 \mathrm{nmol} \mathrm{L} \mathrm{LC}^{-1} \mathrm{AC}$ at excitation wavelength $300 \mathrm{~nm}$ (values are the average of three measurements).

of $5.0 \mathrm{nmol} \mathrm{L}^{-1} \mathrm{AC}$ (Fig. S7). The data obtained showed the highest fluorescence enhancement after 5 min incubation time and thereafter a quenching effect of the PL intensity of the QDs was observed after $10 \mathrm{~min}$, indicating a potential reversible interaction. The results show that although AC can be detected within 5 to $35 \mathrm{~min}$ of incubation time, a shorter incubation time is more effective.

\subsection{Influence of AC on the Fluorescence Behaviour of L-cys-CdSe/ZnS QDs}

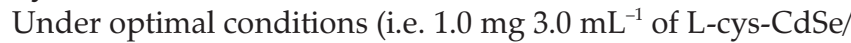
$\mathrm{ZnS}$ QDs and $5 \mathrm{~min}$ as incubation time), the introduction of acetaminophen to the L-cys-CdSe/ZnS QDs solution led to luminescence enhancement (Fig. 6A). This signal enhancement could be due to FRET from analyte which served as donor to QDs. The emission of the acceptor enhances and simultaneously the luminescence of the donor quenches. In this case, due to the weak fluorescence intensity of the $\mathrm{AC}$, the quenching of $\mathrm{AC}$ fluorescence could not be seen in Fig. 6A.

\subsection{Analytical Figures of Merit}

The fluorescence calibration curve of $\mathrm{F}_{-} \mathrm{F}_{0}$ versus AC concentration is illustrated in Fig. 6B, where $\mathrm{F}$ and $\mathrm{F}_{0}$ are the fluorescence intensities of the QDs in the presence and absence of different concentrations of acetaminophen, respectively. A good linear relationship between $\mathrm{F}-\mathrm{F}_{0}$ and $[\mathrm{AC}]$ was found from 3.0-100 $\mathrm{nmol} \mathrm{L}{ }^{-1}$ at an emission wavelength of $595 \mathrm{~nm}\left(\mathrm{r}^{2}=0.99\right)$. Thus, AC can be sensed fluorescently using this method.

The limit of detection (LOD) and quantification (LOQ) are defined as $3 \delta / \mathrm{m}$ and $10 \delta / \mathrm{m}$, respectively, where $\delta$ is the standard deviation of blank measurement (with $\mathrm{n}=10$ ) and $\mathrm{m}$ is the slope of the calibration curve. LOD and LOQ were found to be $1.6 \mathrm{nmol} \mathrm{L}^{-1}$ and $5.3 \mathrm{nmol} \mathrm{L}{ }^{-1}$, respectively. The data thus revealed that the proposed method is suitable for the determination of AC at environmentally relevant levels. The LOD value is lower than the guidance value of $1.3 \times 10^{3} \mathrm{nmol} \mathrm{L}^{-1}$ (or $200 \mathrm{ppb}$ ) for acetaminophen in drinking water given by the Department of Health in Minnesota. ${ }^{40}$

The linear range and detection limit of some other methods for the detection of AC in water samples are summarized in Table 3 for comparison purposes. The results indicate the potential of our sensor for detecting the low concentrations of AC typically present in water samples.

\subsection{Proposed Mechanism}

In theory, Förster resonance energy transfer (FRET) is a nonradiative process and if the emission spectra of target compounds overlap with the absorption spectra of the QDs, energy transfer from the analyte to the QDs could occur upon excitation. ${ }^{44}$ In this study, the absorption spectrum of L-cys-CdSe/ZnS QDs at $430-650 \mathrm{~nm}$ (the highest absorbance is at $580 \mathrm{~nm}$ ) (Fig. 7A) overlaps with the emission spectrum of acetaminophen at 350-650 nm (Fig. 7B). Hence the QDs can absorb the emission energy from acetaminophen resulting in the enhancement of the PL emission of QDs, which was also found experimentally. Scheme 1 shows the enhancement mechanism of the QDs in the presence of the AC via FRET. The electrons of AC can absorb energy resulting in an excited state. When the excited electron of $\mathrm{AC}$ returns to the ground state, the electrons of the QDs can transit to the excited states due to the polar-polar resonance of the donor acceptor. The coupled transitions result in quenching of the fluorescence of donor (AC molecules) and enhancement in fluorescence intensity of the acceptor (L-cys-CdSe/ZnS QDs). ${ }^{45}$

The distance between donor and acceptor is also a critical parameter of FRET processes since FRET depends on the donor quantum yield and will occur over a limited distance (Förster distance $(\AA)$ ) when the overlap between the emission spectrum of the donor and the absorption spectrum of the acceptor is greater than $30 \%$ and the distance is less than $10 \mathrm{~nm} .{ }^{46}$ The Förster distance in the L-cys-CdSe/ZnS QDs-AC system was found to be $6.1 \mathrm{~nm}$ which meets this distance requirement for FRET to occur.

\subsection{Effect of Potential Interfering Analytes on the Fluorescence of L-cys-CdSe/ZnS QDs}

The possible influence of other related pharmaceutical analytes was assessed under optimum conditions of $2 \mathrm{~mL}$ of $1.0 \mathrm{mg} 3.0 \mathrm{~mL}^{-1} \mathrm{~L}$-cys-CdSe/ZnS QDs and $5 \mathrm{~min}$ equilibrium time. Although the photoluminescence intensity of the L-cys-CdSe/ZnS QDs was slightly enhanced in the presence of these pharmaceutical analytes (Fig. 8), it is clear that the prototype QD probe is the most sensitive to AC which provided the highest relative fluorescence intensity whereas the other analytes investigated did not produce as significant a fluorescence enhancement with the QDs (Table S2). These compounds therefore do not interfere significantly with the detection of AC. 

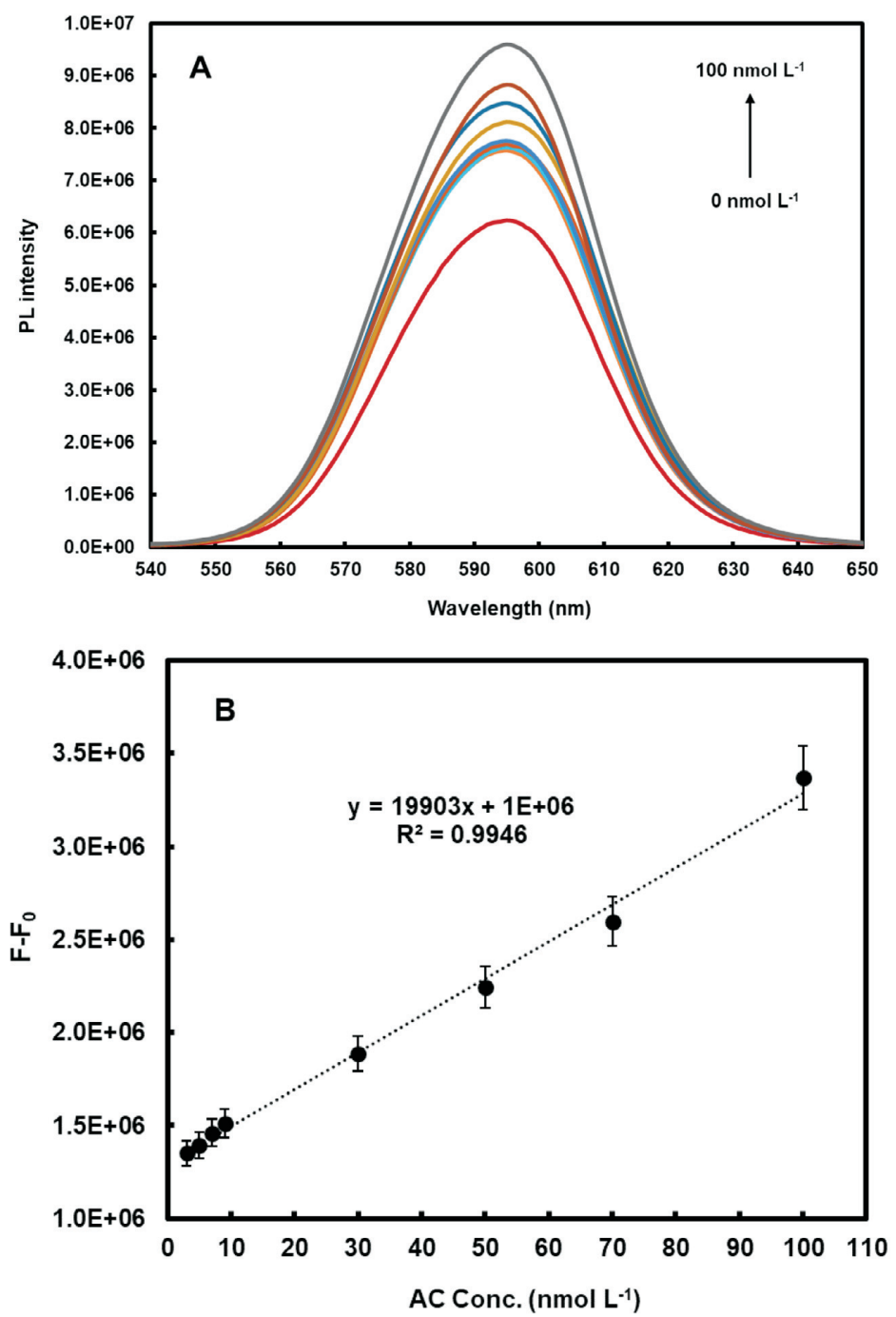

Figure 6 (A) PL 'turn-on' emission spectra for the determination of AC using L-cys-CdSe/ZnS QDs for different concentrations of AC at an excitation wavelength of $300 \mathrm{~nm}$ and (B) linear graph of $\mathrm{F}-\mathrm{F}_{0}$, versus $\mathrm{AC}$ concentration (values are the average of three measurements).

The selectivity of the QDs sensor could be enhanced by the application of a surface molecularly imprinted polymer, for example, should other structurally related compounds be found to interfere with the detection of AC.

\subsection{Sensing Application}

The method was applied to AC in water from a river and tap to investigate the effectiveness of the fluorescence sensor for AC determinations in real samples. As can be seen from Table 4 , the

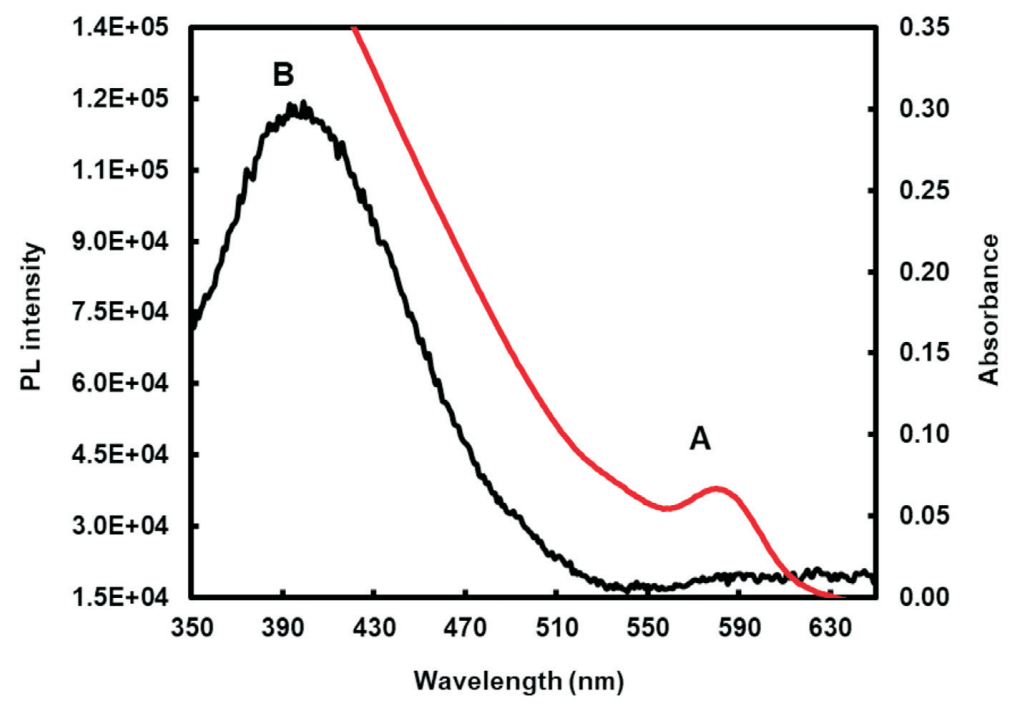

Figure 7 (A) UV-Vis absorption spectrum of L-cys-CdSe/ZnS QDs and (B) fluorescence spectrum of acetaminophen $\left(1.0 \times 10^{-5} \mathrm{~mol} \mathrm{~L}^{-1}\right)$ at excitation wavelength $300 \mathrm{~nm}$. 


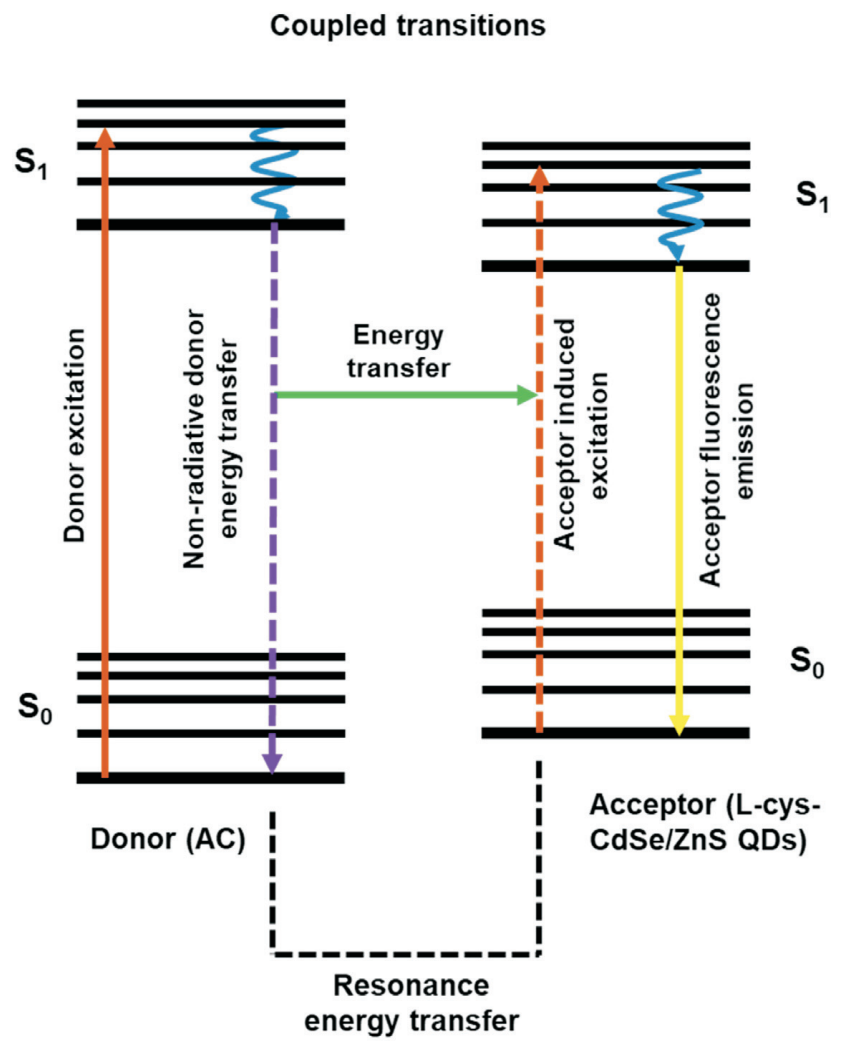

Scheme 1

Fluorescence resonance energy transfer from AC to the L-cys-CdSe/ZnS QDs.

recoveries of different known amounts of AC spiked in both types of water ranged from 95 to $108 \%$ and 90 to $108 \%$, respectively. The fluorescence sensing method developed in this study was thus found to be practical and reliable for the determination of $\mathrm{AC}$ in the aqueous environment.

Regarding the effect of $\mathrm{pH}$, the average three replicate $\mathrm{pH}$ value of L-cys-CdSe/ZnS QDs in Millipore water was 7.6 while after addition of $500 \mu \mathrm{L}$ of tap or river water into $2 \mathrm{~mL}$ of L-cysCdSe/ZnS QDs solution (containing $0.67 \mathrm{mg}$ of L-cys-CdSe/ZnS $\mathrm{QDs}$ ), the $\mathrm{pH}$ values changed to 7.1 and 7.5, respectively. Therefore, no significant $\mathrm{pH}$ changes took place upon addition of real water samples to the L-cys-CdSe/ZnS QDs solution and the effect of sample $\mathrm{pH}$ can consequently be considered negligible. HPLC-MS/MS analysis confirmed that AC was not found in tap and river water samples prior to spiking. Therefore, different amounts of AC were spiked to tap and river water samples in order to investigate the applicability of the method.

\section{Conclusion}

A comparison study was performed by applying three watersoluble L-cys, NAC and GSH functionalized CdSe/ZnS QDs to optimize a QD-ligand system for acetaminophen fluorescence sensing. L-cys-CdSe/ZnS QDs were selected due to their enhanced sensitivity for use as a fluorescence sensor for the

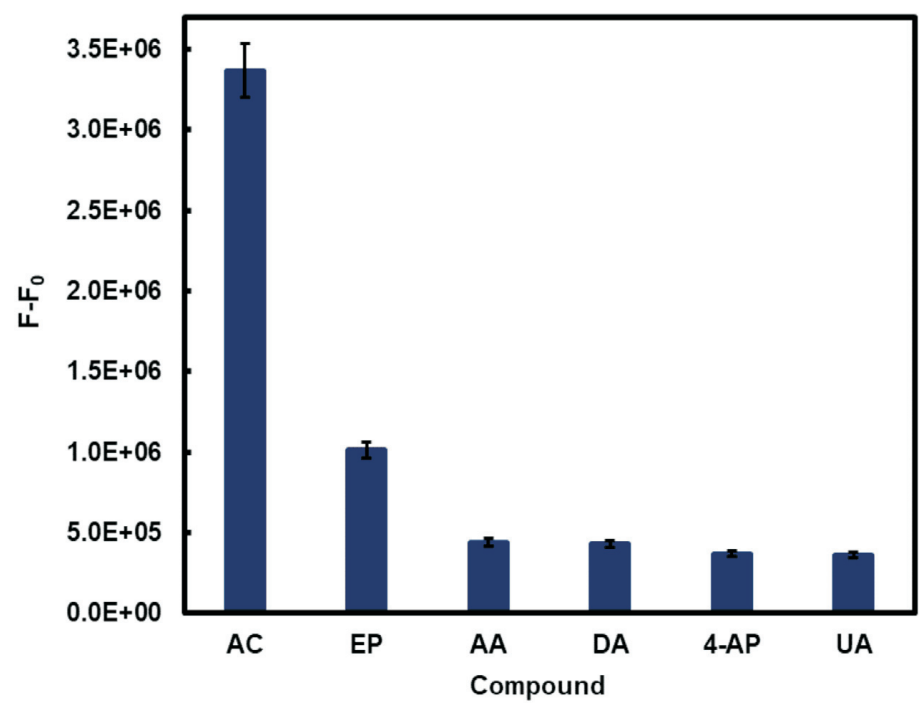

Figure 8 Fluorescence intensity change of $1.0 \mathrm{mg} / 3.0 \mathrm{~mL}$ of L-cys-CdSe/ZnS QDs in the presence of AC and various analytes $\left(100 \mathrm{nmol} \mathrm{L}{ }^{-1}\right)$ at an excitation and emission wavelengths of $300 \mathrm{~nm}$ and $595 \mathrm{~nm}$, respectively. Epinephrine hydrochloride (EP), L-ascorbic acid (AA), uric acid (UA), dopamine hydrochloride (DA), 4-aminophenol (4-AP), (values are the average of three measurements). 
Table 4 Analytical results for the determination of recoveries of $A C$ spiked in tap and river water samples using L-cys-CdSe/ZnS QDs.

\begin{tabular}{lccr}
\hline & $\begin{array}{c}\text { AC spiked } \\
\text { /nmol L }\end{array}$ & $\begin{array}{c}\text { Determined AC } \\
\text { /mean } \pm \text { RSD; } \mathrm{n}=3, \\
\text { nmol L }\end{array}$ & $\begin{array}{c}\text { Recovery } \\
/ \%\end{array}$ \\
\hline Tap water & 10 & $10.1 \pm 0.1$ & 101 \\
& 50 & $54.0 \pm 0.1$ & 108 \\
River water & 100 & $95.0 \pm 0.2$ & 95 \\
& 10 & $10.8 \pm 0.7$ & 108 \\
& 50 & $53.0 \pm 0.1$ & 106 \\
& 100 & $90.0 \pm 0.2$ & 90 \\
\hline
\end{tabular}

determination of acetaminophen in water for the first time. The operating mechanism of the designed probe was based on the fluorescence 'turn-on' mode. Advantages of this method include its simplicity, as well as stability and resistivity of QDs against photobleaching under ambient light conditions. Under optimum conditions, with the addition of acetaminophen to QDs in aqueous solution, the original PL intensity of the QDs was enhanced most likely due to fluorescence resonance energy transfer from analytes to QDs which was found to be linear within the concentration range of $3.0-100 \mathrm{nmol} \mathrm{L}^{-1}$ AC with detection and quantification limits of 1.6 and $5.3 \mathrm{nmol} \mathrm{L}^{-1}$, respectively. The method could therefore be applied to detect $\mathrm{AC}$ at environmentally relevant nanomolar concentrations.

\section{Acknowledgements}

Research funding from the University of Pretoria, the Water Research Commission (Grant K5/2438/1 and K5/2752) as well as the Photonics Initiative of South Africa (Grant PISA-15-DIR-06) is gratefully acknowledged. We also thank the Microscopy and Microanalysis Laboratory of the University of Pretoria, for assistance with microscopy measurements and Wiebke Grote of UP for the XRD measurements.

\section{Supplementary Material}

Supplementary information is provided in the online supplement.

\section{${ }^{5}$ ORCID iDs}

H. Montaseri: iD orcid.org/0000-0002-6433-9594

P.B.C. Forbes: (iD) orcid.org/0000-0003-3453-9162

\section{References}

1 P. Westerhoff, Y. Yoon, S. Snyder and E.C. Wert, Fate of endocrinedisruptor, pharmaceutical, and personal care product chemicals during simulated drinking water treatment processes, Environ. Sci. Technol., 2005, 39, 6649-6663.

2 Y. Ishihama, M. Nakamura, T. Miwa, T. Kajima and N. Asakawa, A rapid method for $\mathrm{pKa}$ determination of drugs using pressureassisted capillary electrophoresis with photodiode array detection in drug discovery, J. Pharm. Sci., 2002, 91, 933-942.

3 A. Achilleos, M. Ines Vasquez Hadjilyra, E. Hapeshi, C. Michael, S.M. Monou, M. and D. Fatta Kassinos, presented in part at the International Conference on the Protection and Restoration of the Environment IX, Kefalonia, Greece, 29 June - 03 July 2008.

4 K.H. Langford and K.V. Thomas, Determination of pharmaceutical compounds in hospital effluents and their contribution to wastewater treatment works, Environ. Int., 2009, 35, 766-770.

5 M. Crane, C. Watts and T. Boucard, Chronic aquatic environmental risks from exposure to human pharmaceuticals, Sci. Total Environ., 2006, 367, 23-41.

6 M.M. Schultz, E.T. Furlong, D.W. Kolpin, S.L. Werner, H.L. Schoenfuss, L.B. Barber, V.S. Blazer, D.O. Norris and A.M. Vajda, Antidepressant pharmaceuticals in two US effluent-impacted streams: occurrence and fate in water and sediment, and selective uptake in fish neural tissue, Environ. Sci. Technol., 2010, 44, 1918-1925. 
7 S. Matongo, G. Birungi, B. Moodley and P. Ndungu, Occurrence of selected pharmaceuticals in water and sediment of Umgeni River, KwaZulu-Natal, South Africa, Environ. Sci. Pollut. Res. Int., 2015, 22, 10298-10308.

8 S. Matongo, G. Birungi, B. Moodley and P. Ndungu, Pharmaceutical residues in water and sediment of Msunduzi River, Kwazulu-Natal, South Africa, Chemosphere, 2015, 134, 133-140.

9 O. K'Oreje, K.L. Vergeynst, D. Ombaka, P. De Wispelaere, M. Okoth $\mathrm{H}$. Van Langenhove and K. Demeestere, Occurrence patterns of pharmaceutical residues in wastewater, surface water and groundwater of Nairobi and Kisumu city, Kenya, Chemosphere, 2016, 149, 238-244.

10 M. Gros, M. Petrovic and D. Barcelo, Development of a multi-residue analytical methodology based on liquid chromatography-tandem mass spectrometry (LC-MS/MS) for screening and trace level determination of pharmaceuticals in surface and wastewaters, Talanta 2006, 70, 678-690.

11 M.J. Gómez, M.J.M. Bueno, S. Lacorte, A.R. Fernández-Alba and A. Agüera, Pilot survey monitoring pharmaceuticals and related compounds in a sewage treatment plant located on the Mediterranean coast, Chemosphere, 2007, 66, 993-1002.

12 P.H. Roberts and K.V. Thomas, The occurrence of selected pharmaceuticals in wastewater effluent and surface waters of the lower Tyne catchment, Sci. Total Environ., 2006, 356, 143-153.

13 Ş. Dinç, Ö.A. Dönmez, B. Aş̧̧ı and A.E. Bozdoğan, Chromatographic and chemometrics-assisted spectrophotometric methods for the simultaneous determination of allobarbital, adiphenine hydrochloride, and paracetamol in suppository, J. Liq. Chromatogr. Relat. Technol., 2014, 37, 560-571.

14 B. Zheng, C. Li, Y. Li, Y. Gu, X. Yan, L. Tang, R. Chen and Z. Zhang, One-step electrochemical preparation of a reduced graphene oxide/ poly (sulfosalicylic acid) nanocomposite film for detection of acetaminophen and its application in human urine and serum studies, Anal. Methods, 2015, 7, 8248-8254.

15 A. Méndez-Albores, C. Tarín, G. Rebollar-Pérez, L. DominguezRamirez and E. Torres, Biocatalytic spectrophotometric method to detect paracetamol in water samples, J. Environ. Sci. Health A., 2015, 50, 1046-1056.

16 D. Easwaramoorthy, Y.-C. Yu and H.-J. Huang, Chemiluminescence detection of paracetamol by a luminol-permanganate based reaction, Anal. Chim. Acta, 2001, 439, 95-100.

17 L. Yuan, W. Lin, K. Zheng, L. He and W. Huang, Far-red to near infrared analyte-responsive fluorescent probes based on organic fluorophore platforms for fluorescence imaging, Chem. Soc. Rev., 2013, 42, 622-661.

18 Q. Liu, Q. Zhou and G. Jiang, Nanomaterials for analysis and monitoring of emerging chemical pollutants, Trends Anal. Chem., 2014, 58, $10-22$.

19 R. Gill, M. Zayats and I. Willner, Semiconductor quantum dots for bioanalysis, Angew. Chem. Int. Ed., 2008, 47, 7602-7625.

20 Y. Su, Y. He, H. Lu, L. Sai, Q. Li, W. Li, L. Wang, P. Shen, Q. Huang and C. Fan, The cytotoxicity of cadmium based, aqueous phase-synthesized, quantum dots and its modulation by surface coating, Biomaterials, 2009, 30, 19-25.

21 H. Xu, R. Miao, Z. Fang and X. Zhong, Quantum dot-based "turn-on" fluorescent probe for detection of zinc and cadmium ions in aqueous media, Anal. Chim. Acta, 2011, 687, 82-88.

22 S. Ithurria, P. Guyot-Sionnest, B. Mahler and B. Dubertret, $\mathrm{Mn}^{2+}$ as a radial pressure gauge in colloidal core/shell nanocrystals, Phys. Rev. Lett., 2007, 99, 265501.

23 K.T. Yong, W.C. Law, I. Roy, Z. Jing, H. Huang, M.T. Swihart and P.N. Prasad, Aqueous phase synthesis of CdTe quantum dots for biophotonics, J. Biophotonics., 2011, 4, 9-20.

24 R.K. Ratnesh and M.S. Mehata, Investigation of biocompatible and protein sensitive highly luminescent quantum dots/nanocrystals of CdSe, CdSe/ZnS and CdSe/CdS, Spectrochim. Acta A. Mol. Biomol. Spectrosc., 2017, 179, 201-210.

25 A.J. Nozik, M.C. Beard, J.M. Luther, M. Law, R.J. Ellingson and J.C. Johnson, Semiconductor quantum dots and quantum dot arrays and applications of multiple exciton generation to third-generation photovoltaic solar cells, Chem. Rev., 2010, 110, 6873-6890.

26 H. Huang and J.-J. Zhu, The electrochemical applications of quantum dots, Analyst, 2013, 138, 5855-5865.
$27 \mathrm{H}$. Montaseri and P.B. Forbes, A triclosan turn-ON fluorescence sensor based on thiol-capped core/shell quantum dots, Spectrochim. Acta A. Mol. Biomol. Spectrosc., 2018, 204, 370-379.

28 H. Du, R.C.A. Fuh, J. Li, L. A. Corkan and J.S. Lindsey, PhotochemCAD: a computer-aided design and research tool in photochemistry, Photochem. Photobiol., 1998, 68, 141-142.

29. A. Bagga, P.K. Chattopadhyay and S. Ghosh, Stokes shift in quantum dots: origin of dark exciton, Physics of Semiconductor Devices, 2007. IWPSD 2007. International Workshop on, IEEE, 2007, pp. 876-879.

30 O. Adegoke, T. Nyokong and P.B.C. Forbes, Structural and optical properties of alloyed quaternary CdSeTeS core and CdSeTeS/ZnS core-shell quantum dots, J. Alloys Compd., 2015, 645, 443-449.

31 W.W. Yu, L. Qu, W. Guo and X. Peng, Experimental determination of the extinction coefficient of CdTe, CdSe, and CdS nanocrystals, Chem. Mater., 2003, 15, 2854-2860.

32 B.O. Dabbousi, J. Rodriguez-Viejo, F.V. Mikulec, J.R. Heine, H. Mattoussi, R. Ober, K.F. Jensen and M.G. Bawendi, (CdSe) ZnS core-shell quantum dots: synthesis and characterization of a size series of highly luminescent nanocrystallites, J. Phys. Chem. B., 1997, 101, 9463-9475.

33 O. Adegoke, M.-W. Seo, T. Kato, S. Kawahito and E.Y. Park, Gradient band gap engineered alloyed quaternary/ternary CdZnSeS/ZnSeS quantum dots: an ultrasensitive fluorescence reporter in a conjugated molecular beacon system for the biosensing of influenza virus RNA, J. Mater. Chem. B., 2016, 4, 1489-1498.

34 A.V. Baranov, Y.P. Rakovich, J.F. Donegan, T.S. Perova, R.A. Moore, D.V. Talapin, A.L. Rogach, Y. Masumoto and I. Nabiev, Effect of ZnS shell thickness on the phonon spectra in CdSe quantum dots, Phys. Rev. B., 2003, 68, 165306.

35 M. Klein, F. Hache, D. Ricard and C. Flytzanis, Size dependence of electron-phonon coupling in semiconductor nanospheres: the case of CdSe, Phys. Rev. B., 1990, 42, 11123-11132.

36 S.N. Alam, N. Sharma and L. Kumar, Synthesis of graphene oxide (GO) by modified hummers method and its thermal reduction to obtain reduced graphene oxide (rGO), Graphene, 2017, 6, 1-18.

37 D.K. Gupta, M. Verma, K. Sharma and N.S. Saxena, Synthesis, characterization and optical properties of $\mathrm{CdSe} / \mathrm{CdS}$ and $\mathrm{CdSe} / \mathrm{ZnS}$ core-shell nanoparticles, Indian J. Pure Appl. Phys., 2017, 55, 113-121.

38 C.-T. Cheng, C.-Y. Chen, C.-W. Lai, W.-H. Liu, S.-C. Pu, P.-T. Chou, Y.-H. Chou and H.-T. Chiu, Syntheses and photophysical properties of type-II CdSe/ZnTe/ZnS (core/shell/shell) quantum dots, J. Mater. Chem., 2005, 15, 3409-3414.

39 M. Koneswaran and R. Narayanaswamy, L-Cysteine-capped ZnS quantum dots based fluorescence sensor for $\mathrm{Cu}^{2+}$ ion, Sens. Actuators B. Chem., 2009, 139, 104-109.

40 Toxicological Summary for: Acetaminophen. (2010). Retrieved from www.health.state.mn.us/divs/eh/risk/guidance/dwec/sumacetamin. pdf, accessed February 2018.

41 F.A. Gorla, E.H. Duarte, E.R. Sartori and C.R.T. Tarley, Electrochemical study for the simultaneous determination of phenolic compounds and emerging pollutant using an electroanalytical sensing system based on carbon nanotubes/surfactant and multivariate approach in the optimization, Microchem. J., 2016, 124, 65-75.

42 C.D.S. Pereira, L.A. Maranho, F.S. Cortez, F.H. Pusceddu, A.R. Santos, D.A. Ribeiro, A. Cesar and L.L. Guimarães, Occurrence of pharmaceuticals and cocaine in a Brazilian coastal zone, Sci. Total Environ., 2016, 548, 148-154.

43 L. Capka, P. Lacina and M. Vavrova, Development and application of SPE/CZE method for detection and determination of selected non-steroidal anti-inflammatory drugs in wastewater, Fresen. Environ. Bull., 2012, 21, 3312-3317.

44 K.E. Sapsford, L. Berti and I.L. Medintz, Materials for fluorescence resonance energy transfer analysis: beyond traditional donor-acceptor combinations, Angew. Chem. Int. Ed., 2006, 45, 4562-4589.

45 X. Wang, J. Yu, Q. Kang, D. Shen, J. Li and L. Chen, Molecular imprinting ratiometric fluorescence sensor for highly selective and sensitive detection of phycocyanin, Biosens. Bioelectron., 2016, 77, 624-630.

46 M. Elangovan, R. Day and A. Periasamy, Nanosecond fluorescence resonance energy transfer-fluorescence lifetime imaging microscopy to localize the protein interactions in a single living cell, J. Microsc., 2002, 205, 3-14. 


\section{Supplementary material to:}

H. Montaseri, O. Adegoke and P.B.C. Forbes,

Development of a Thiol-capped Core/Shell Quantum Dot Sensor for Acetaminophen, S. Afr. J. Chem., 2019, 72, 108-117. 


\section{Development of a thiol-capped core/shell quantum dot sensor for acetaminophen}

Hanieh Montaseri, Oluwasesan Adegoke, Patricia B.C. Forbes*

Department of Chemistry, Faculty of Natural and Agricultural Sciences, University of

Pretoria, Lynnwood Road, Pretoria 0002, South Africa

* Corresponding author. Tel.: +27 124205426.

E-mail address: patricia.forbes@up.ac.za (P.B.C. Forbes). 


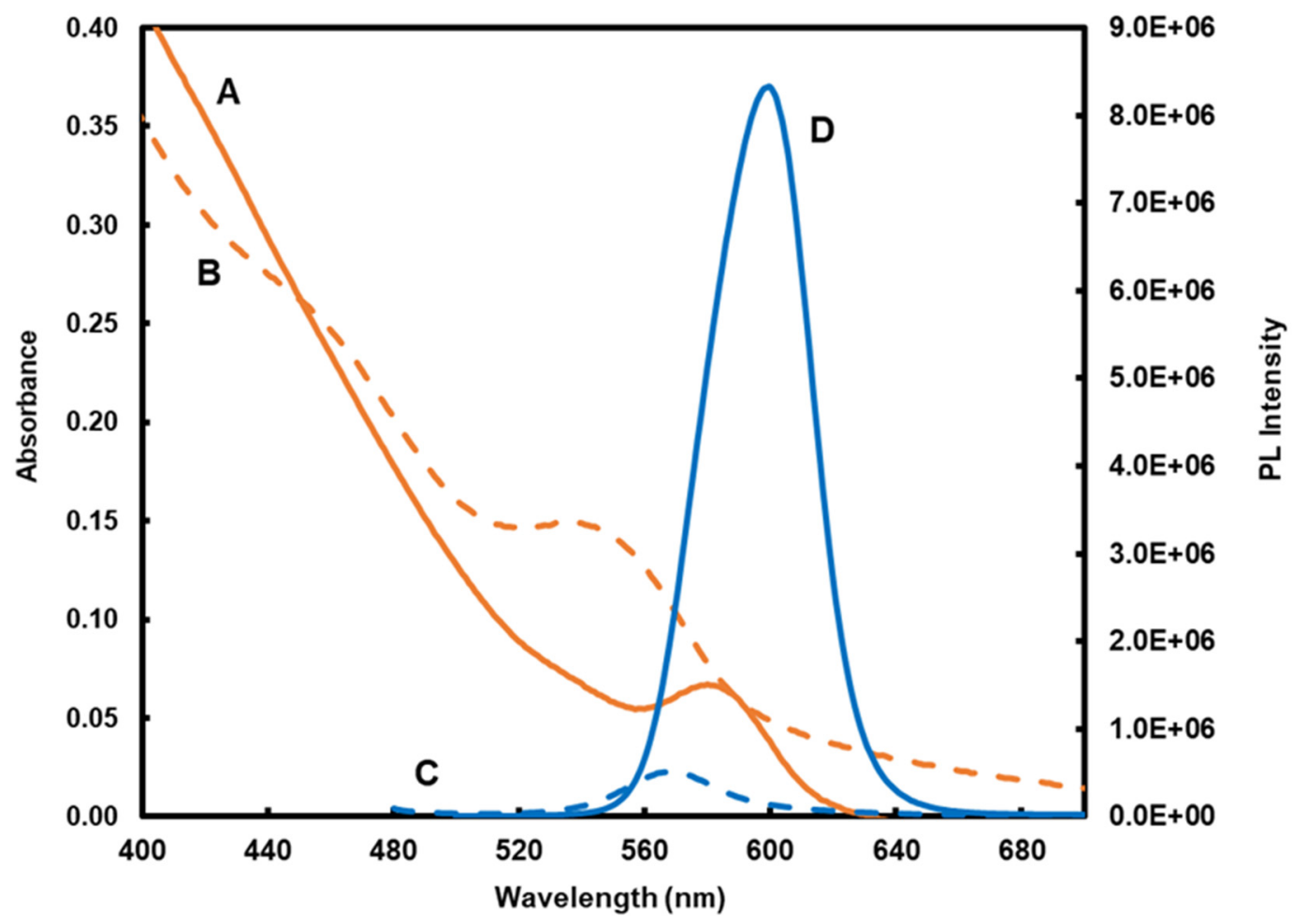

Fig. S1.Absorption spectra of the water-soluble (A) L-cys-capped CdSe/ZnS QDs; (B) L-cys-CdSe QDs in Millipore water;(C) PL emission spectra of the water-soluble L-cys-capped CdSe QDs at excitation wavelength $470 \mathrm{~nm}$;(D) L-cys-capped CdSe/ZnS QDs at excitation wavelength $410 \mathrm{~nm}$ measured in Millipore water.
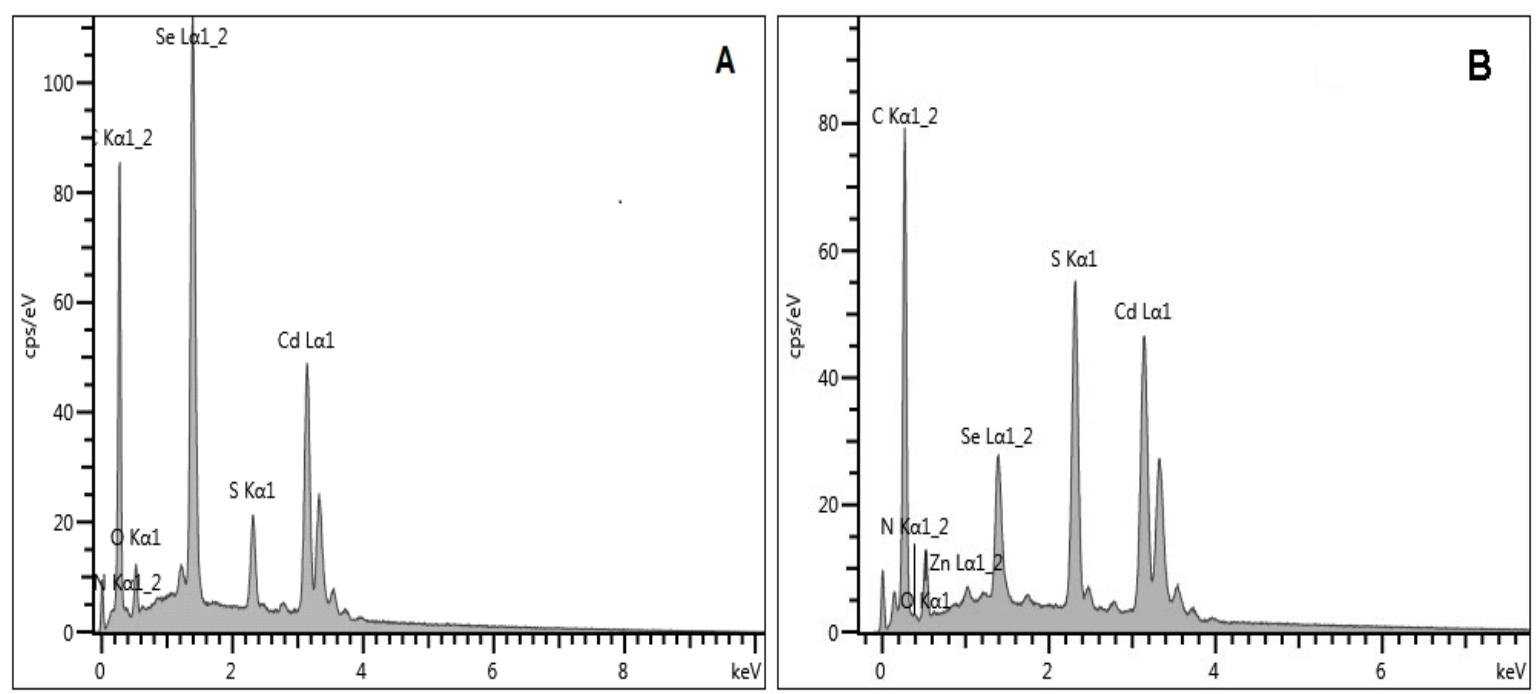

Fig. S2.EDS spectra of (A) L-cys-CdSe QDs and (B) L-cys-CdSe/ZnS QDs. 


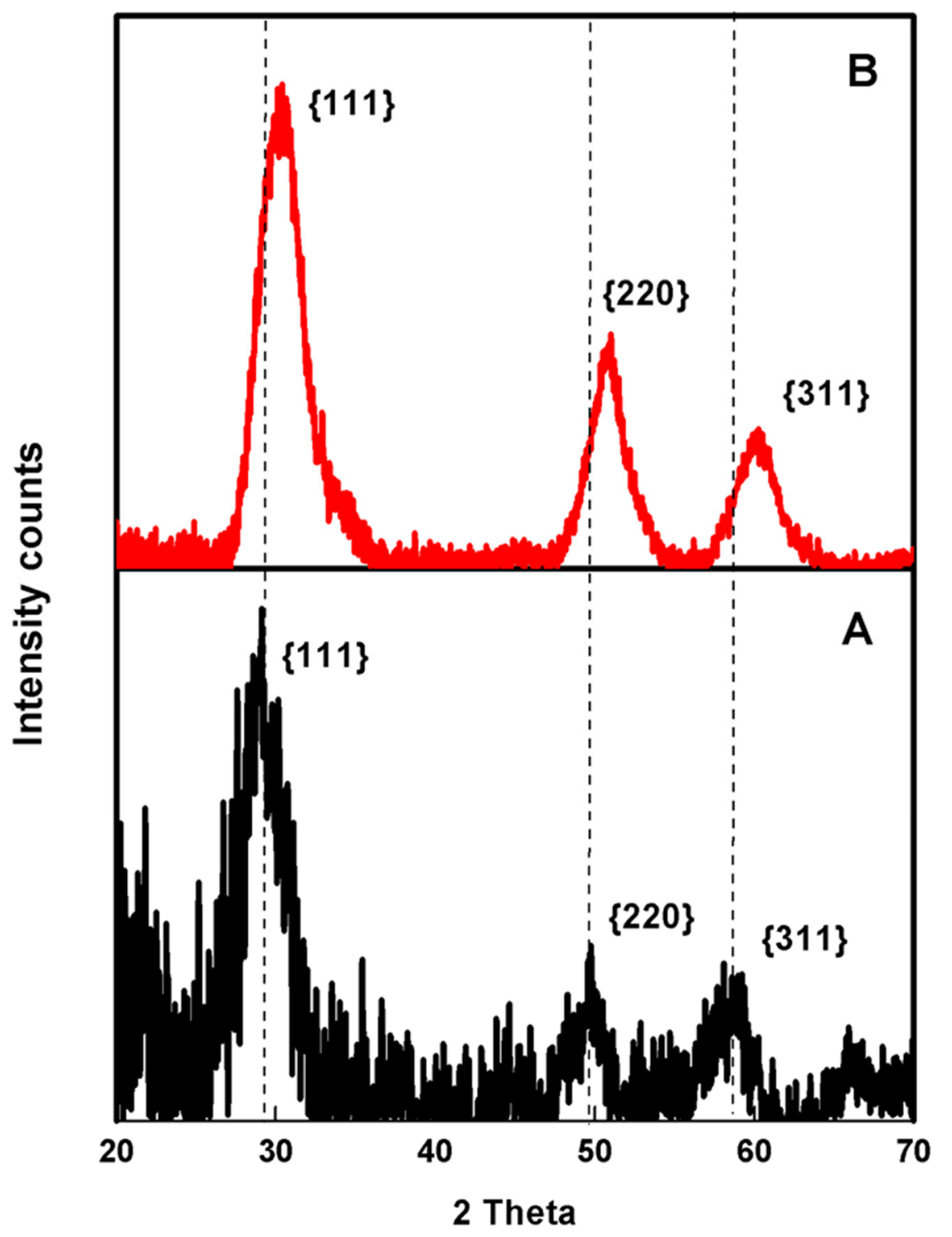

Fig. S3.Powder XRD pattern of the (A) L-cys-CdSe and (B) L-cys-CdSe/ZnS QDs. 


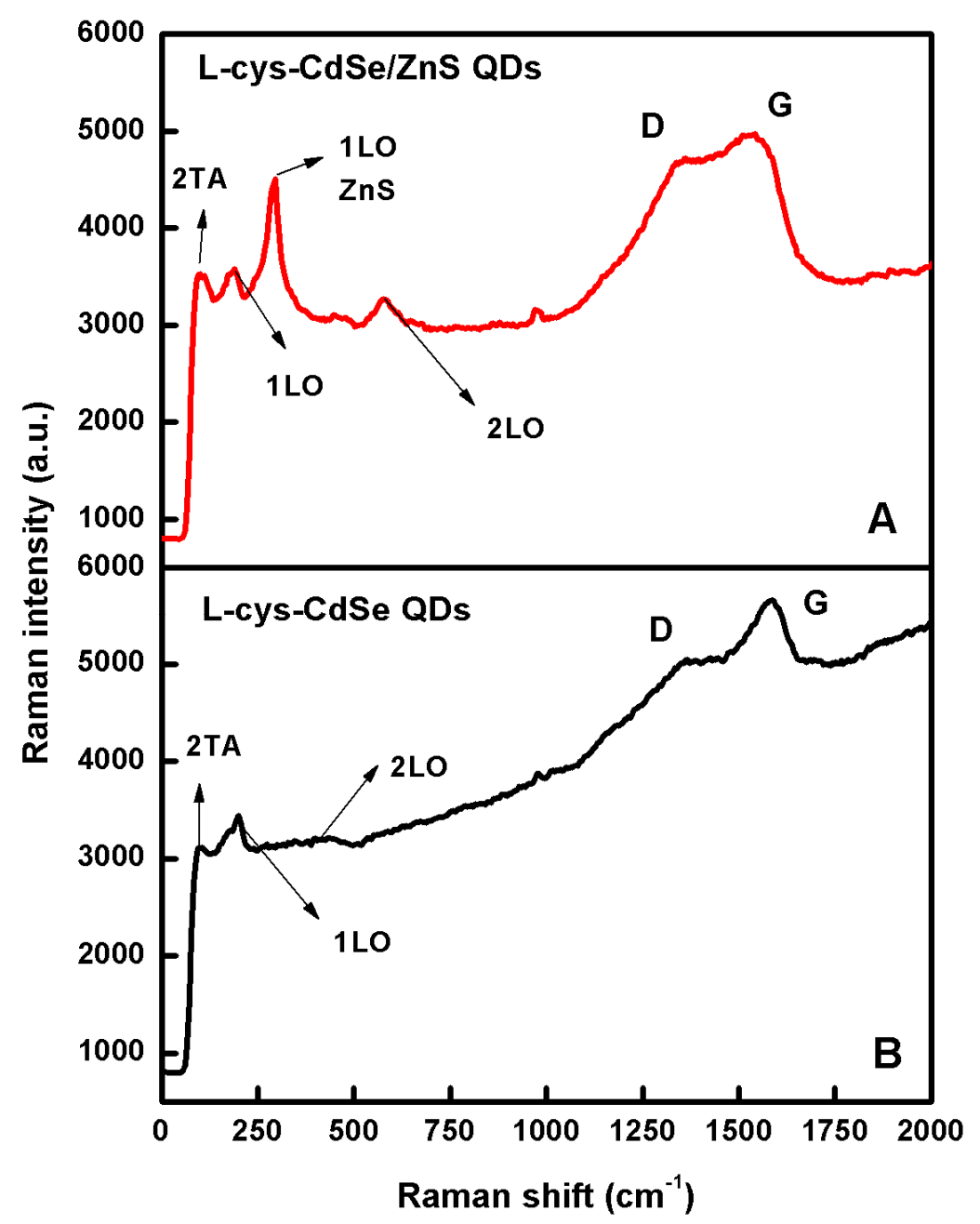

Fig. S4.Raman spectra of (A) L-cys-CdSe/ZnS QDs and (B) L-cys-CdSe QDs. 


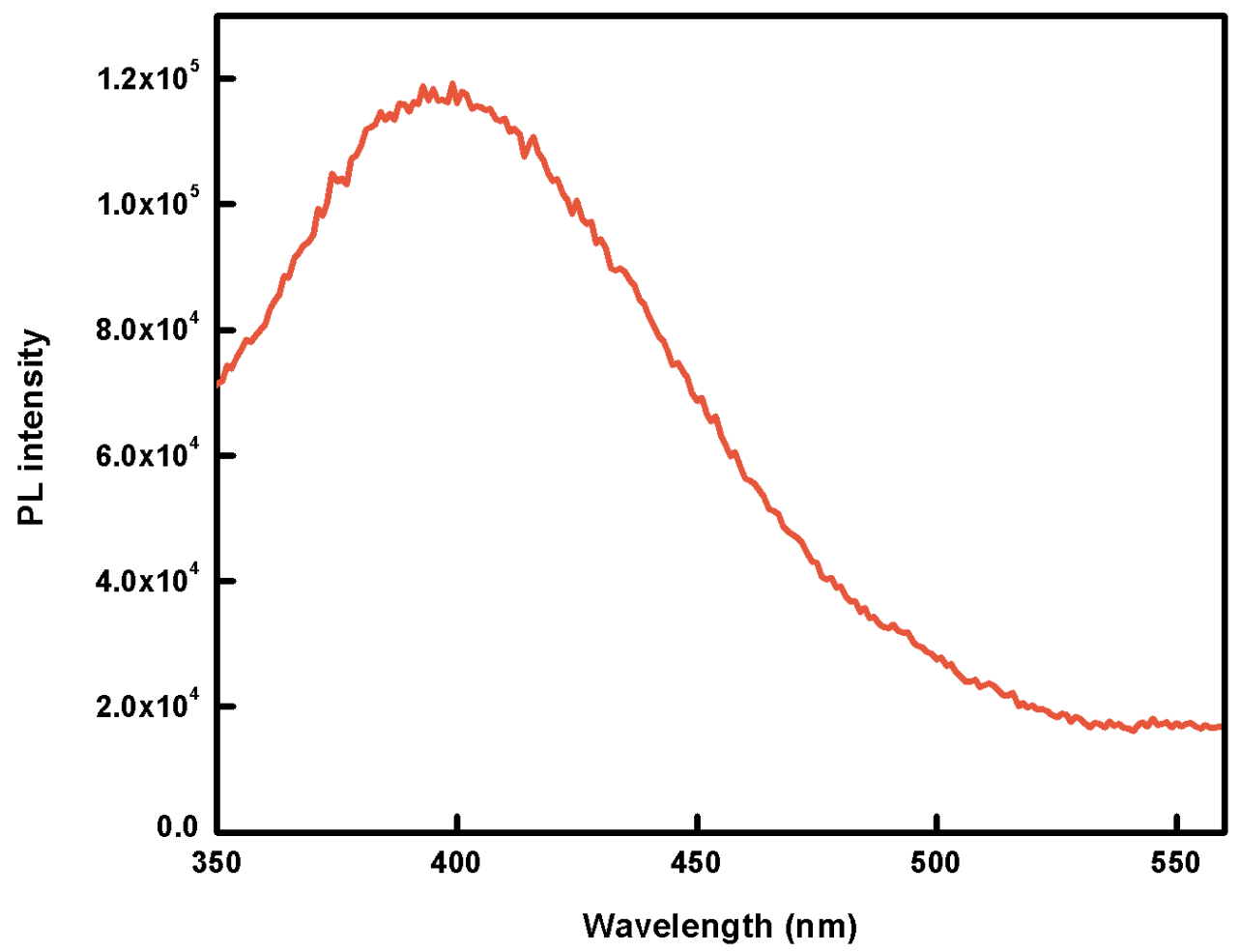

Fig. S5.Florescence spectrum of acetaminophen in water $\left(1.0 \times 10^{-5} \mathrm{~mol} \mathrm{~L}^{-1}\right)$ at an excitation wavelength of $300 \mathrm{~nm}$.

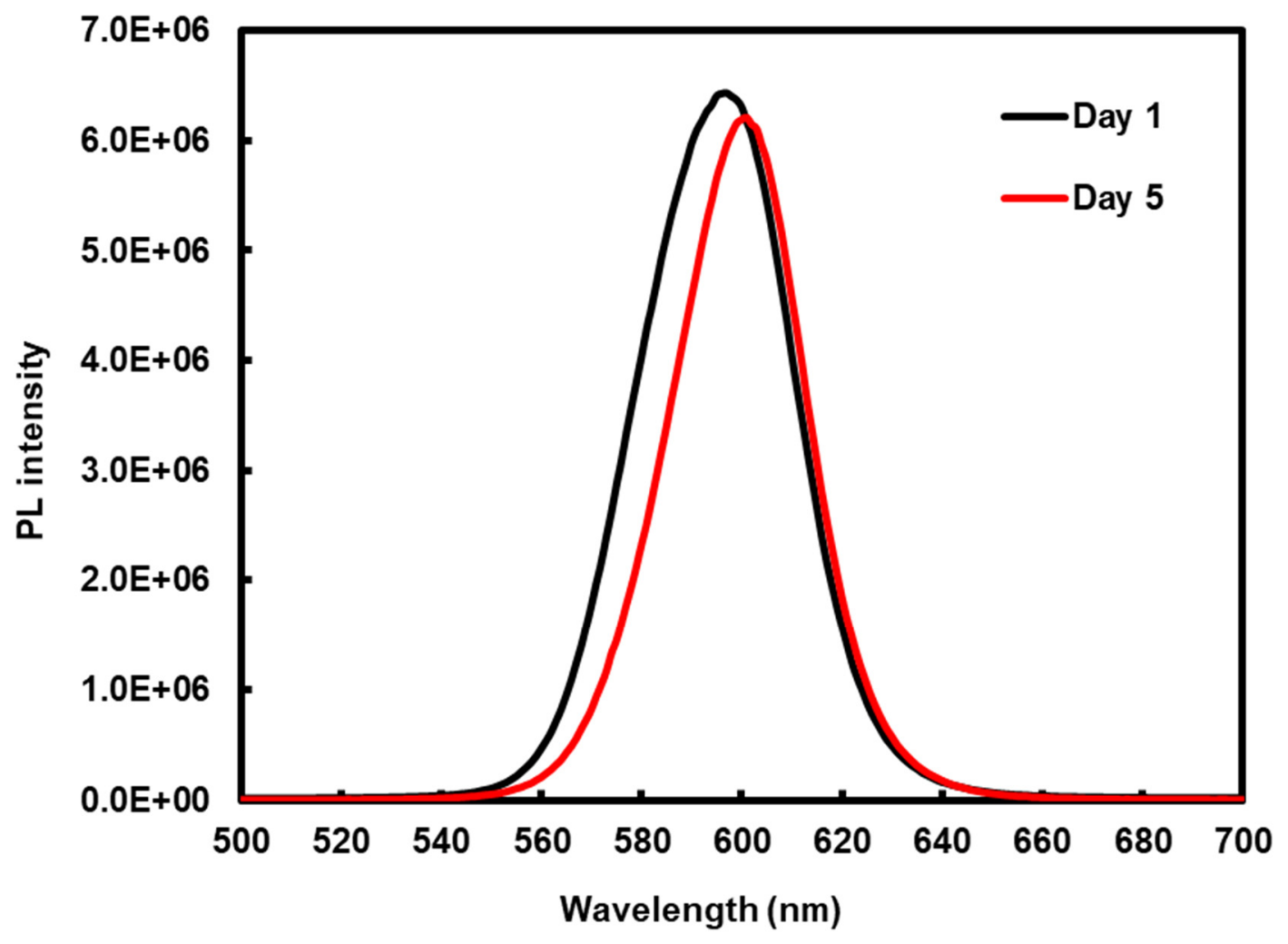

Fig. S6.PL stability of the hydrophilic QDs measured before and after 5 days of exposure to ambient light (300 $\mathrm{nm}$ excitation). 


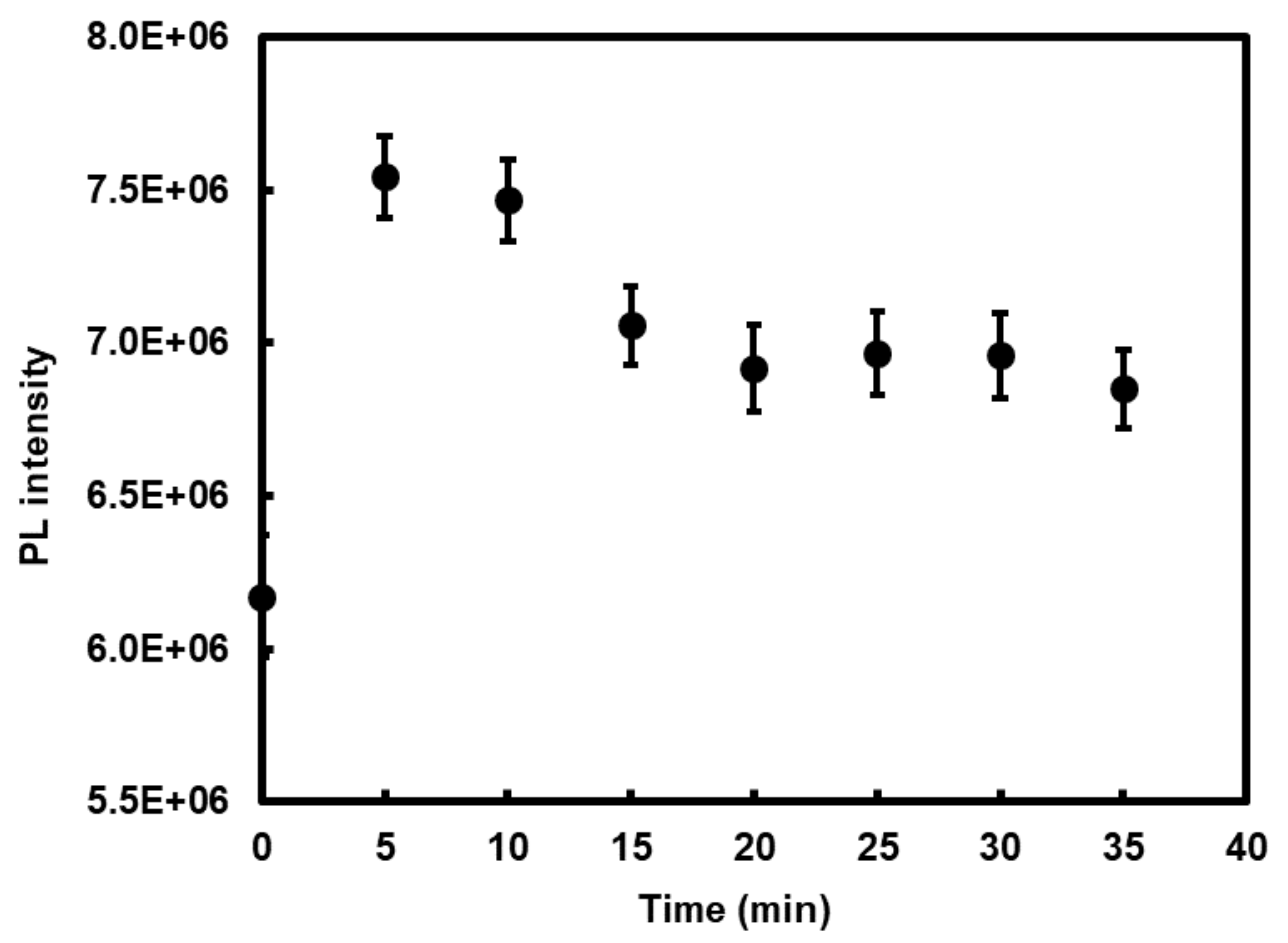

Fig. S7.Effect of interaction time on the fluorescence intensity of the L-cys capped CdSe/ZnS QD-AC system at an excitation wavelength of $300 \mathrm{~nm}$ (values are the average of three measurements). 

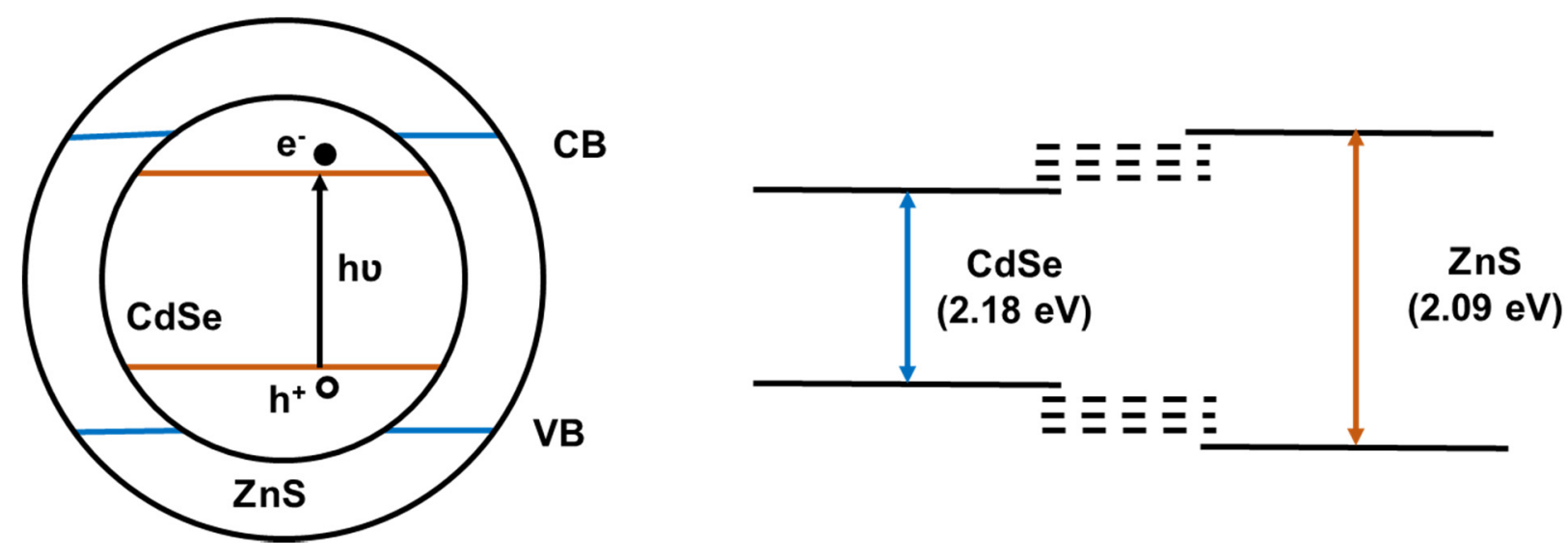

Scheme S1. Schematic representation of the influence of the larger band gap induced by the ZnS shellover the core QDs.

\section{Table S1}

IR absorption bands of the L-cys-CdSe and L-cys-CdSe/ZnS QDs.

\begin{tabular}{cc}
\hline Functional group & IR band $\left(\mathrm{cm}^{-1}\right)$ \\
\hline$-\mathrm{COO}-$ & $\begin{array}{c}1550-1600 \text { (strong) } \\
1400 \text { (medium) }\end{array}$ \\
$-\mathrm{OH}$ & $3000-3500$ \\
$-\mathrm{NH}_{2}$ & $2900-3420$ \\
$\mathrm{C}-\mathrm{S}$ & $600-800$ \\
$\mathrm{~S}-\mathrm{H}$ & $2550-2750$ \\
\hline
\end{tabular}




\section{Table S2}

Chemical structures of potentially interfering analytes.

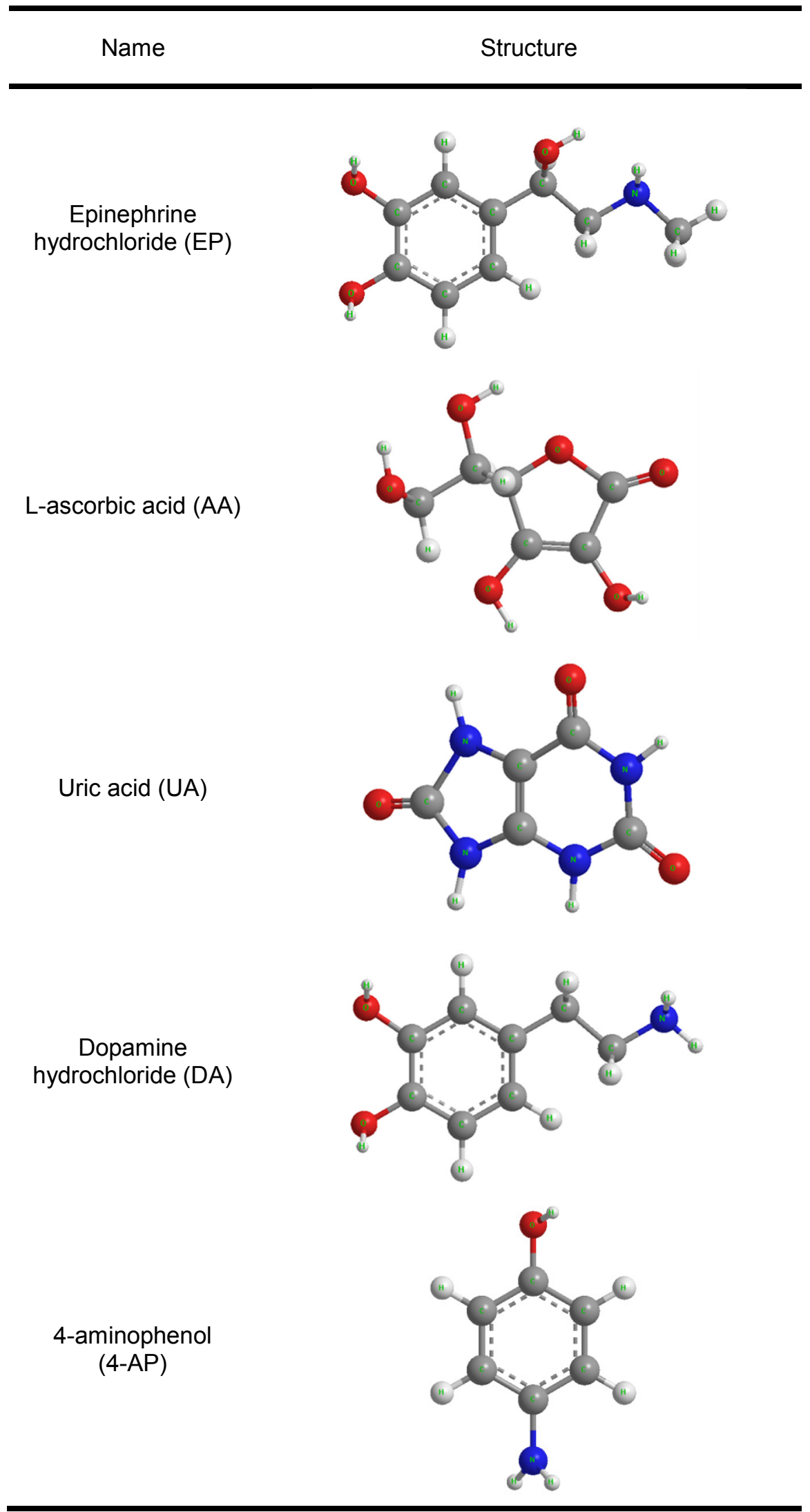

